\title{
Atomistic near-field nanoplasmonics: reaching atomic-scale resolution in nanooptics
}

\author{
M. Barbry, ${ }^{\dagger}$ P. Koval, ${ }^{\dagger}$ F. Marchesin, ${ }^{\dagger}$ R. Esteban,${ }^{\dagger}$ A. G. Borisov, ${ }^{\ddagger}$ J. \\ Aizpurua, ${ }^{*, \dagger}$ and D. Sánchez-Portal*,† \\ †Centro de Física de Materiales, Centro Mixto CSIC-UPV/EHU and Donostia \\ International Physics Center (DIPC), Paseo Manuel de Lardizabal 5, 20018 Donostia-San \\ Sebastián, Spain \\ $\ddagger$ Institut des Sciences Moléculaires d'Orsay ISMO, UMR 8214 CNRS-Université \\ Paris-Sud, Bât. 351, Université Paris-Sud, 91405 Orsay Cedex, France \\ E-mail: aizpurua@ehu.es; sqbsapod@ehu.es
}

\begin{abstract}
Electromagnetic field localization in nanoantennas is one of the leitmotivs that drives the development of plasmonics. The near-fields in these plasmonic nanoantennas are commonly addressed theoretically within classical frameworks that neglect atomic-scale features. This approach is often appropriate since the irregularities produced at the atomic scale are typically hidden in far-field optical spectroscopies. However, a variety of physical and chemical processes rely on the fine distribution of the local fields at this ultraconfined scale. We use time-dependent density functional theory and perform atomistic quantum mechanical calculations of the optical response of plasmonic nanoparticles, and their dimers, characterized by the presence of crystallographic planes, facets, vertices, and steps. Using sodium clusters as an example, we show that the atomistic details of the nanoparticles morphologies determine the presence of subnanometric near-field hot spots that are further enhanced by the action of
\end{abstract}


the underlying nanometric plasmonic fields. This situation is analogue to a self-similar nanoantenna cascade effect, scaled down to atomic dimensions, and it provides new insights into the limits of field enhancement and confinement, with dramatic implications in the optical resolution of field-enhanced spectroscopies and microscopies.

\section{Keywords}

Plasmonic nanoantennas, optical response, TDDFT, DFT ab-initio calculations, field enhancement

Metallic nanoparticles are key in the development of nanooptics. The ability of the conduction electrons to collectively oscillate produces surface charge density oscillations in nanoparticles, so-called surface plasmons, that couple very efficiently to light, producing subwavelength localization and large enhancement of the optical fields induced at the nanoparticles. ${ }^{1-6}$ Nanooptics with localized surface plasmons has thus boosted a variety of technological applications in which the intense electromagnetic fields can assist in enhancing the signal from vibrational spectroscopies, ${ }^{7-10}$ improving the performance of solar cells, ${ }^{11,12}$ optimizing the active control of nanodevices, ${ }^{13-16}$ or implementing noninvasive thermotherapies in medicine, ${ }^{17}$ among others. In all these applications, the optical response that determines the properties of plasmonic surface modes is typically determined in the framework of classical electrodynamics, by solving Maxwell's equations for a particular material, shape, size and environment. In this way, for instance, plasmonic modes of spherical nanoparticles, ${ }^{18,19}$ nanoshells, ${ }^{20}$ nanorings,${ }^{21}$ nanorods, ${ }^{22-25}$ nanostars, ${ }^{26,27}$ dimers,${ }^{3,28,29}$ or particle oligomers ${ }^{30,31}$ have been routinely estimated during the last years. The mode volumes typically reached in these structures are in the range of some tens of nanometers, and the actual degree of their field confinement is determined by the morphology of the nanostructure (curvature, thickness, interaction between different particles,...), ${ }^{5,32-34}$ The effective squeezing of electromagnetic energy into these nanometric dimensions has triggered out referring to plasmonic nanostructures as optical nanoantennas. ${ }^{35,36}$ 
As nanotechnology reaches a control of nanoarchitectures at scales of the order of the nanometer and even subnanometer, ${ }^{37-41}$ nanooptics is called to face new regimes of interaction, where the atomic scale needs to be considered to correctly determine the optical response of the nanosystem. Optical processes at the atomic scale can be critical in many branches of nanoscience such as in field-enhanced photochemistry, ${ }^{42-45}$ in single molecule spectroscopy, ${ }^{8,46-49}$ or in electronics at optical frequencies. ${ }^{38,50-53}$

Most of these situations require a complete theoretical framework that accounts for the quantum nature of the electrons in their interaction with light. Time-dependent Density Functional Theory (TDDFT) ${ }^{54,55}$ provides the adequate framework to tackle the optical response of plasmonic nanoantennas where the complex nonlocal screening, ${ }^{56,57}$ the smooth electronic density profile at the metal interface, ${ }^{58}$ quantum size effects, ${ }^{59-61}$ and electron tunneling across metallic nanogaps ${ }^{62,63}$ can be properly considered. Furthermore, an atomistic description of the nanostructures ${ }^{64}$ can address the effect in the optical response of atomic-scale features at the surfaces such as the presence of protruding atoms, steps, vertices, or edges at the contact of crystallographic planes. Although these atomic-scale effects might be sometimes masked in experimental far-field techniques, they are very relevant in spectroscopy techniques that directly rely on the ultrafine details of the near-field intensity and distribution.

Here we theoretically show how the atomic features at the surfaces of plasmonic nanoparticles do localize electromagnetic fields down to atomic-scale dimensions showing resonant (plasmonic) and nonresonant (lightning rod effect) field enhancements that alter the standard conception of localization of plasmonic fields. With help of our TDDFT calculations, we show near-field maps of plasmonic nanoparticles and their dimers, which produce atomicscale hot spots superimposed to the plasmonic resonances. These are the atomic-scale analogue of the lightning rod effect, ${ }^{65-67}$ which can also be understood as the atomistic limit of the classical field divergence at an infinitely sharp metallic tip. Indeed, the sharpest possible structure would be set by a vertex ending with a single atom. The quantum description 
of such system, where the non-local screening of conduction electrons is included, ${ }^{68}$ sets a limit for the attainable field enhancement. However, when this effect is combined with the overall field enhancement given by a plasmon resonance, we show here that very intense and localized atomic-scale hot spots can be obtained. The presence of these hot spots has been proposed and even exploited experimentally in subnanometer-resolved surface-enhanced optical spectroscopies. ${ }^{49,69-72}$ An accurate description of the physical properties of atomic-scale local near-fields has been elusive to date due to the limitations of classical and quantum descriptions that, even if capable of correctly reporting the main trends of the response, are typically based on strong approximations either on the electromagnetic boundary conditions or the atomic structure of surfaces. Our calculations provide a faithful description of the actual near-field induced in metallic interfaces considering realistic atomic structures, unambiguously resolving the near-field features at the hot spots with unprecedented resolution and detail. We can thus quantify the level of confinement of the near fields relevant in complex photochemical processes and near-field spectroscopies. Our results indicate that, at the vertices and edges formed at the contact of different facets, it is possible to localize plasmons with subnanometric resolution and beat the typical plasmonic confinement imposed by the nanoparticle size. Thus, similar to cluster and surface science where the distinct electronic properties of atoms at steps and edges between crystallographic facets have been extensively considered, e.g., in the context of reactivity, ${ }^{73-76}$ the atomic-scale structure of plasmonic interfaces appears as a new arena of research. This extreme plasmon-localization might allow to implement and exploit novel probes, capable to provide ultraresolution and reach access to information of single molecules.

We apply our atomistic calculations to obtain the optical response and the near-field distribution in a single $\mathrm{Na}_{380}$ cluster, as well as in dimers formed by two $\mathrm{Na}_{380}$ clusters. This is the largest cluster size for which the global minimum icosahedral symmetry (as described using an effective Murrell-Mottram potential ${ }^{77}$ ) is available. ${ }^{78}$ We took this structure ${ }^{79}$ as a starting point for further ab initio relaxation using density functional theory (DFT), as 
implemented in the SIESTA code, ${ }^{80,81}$ within the local density approximation ${ }^{82}$ (LDA). The resulting atomic structure of the $\mathrm{Na}_{380}$ cluster is very similar to the initial one, although it has a higher density due to the well-known underestimation of the sodium lattice parameter by LDA. ${ }^{83}$ This result confirms that the selected structure is stable and at least corresponds to a local minimum of the DFT energy landscape of $\mathrm{Na}_{380}$. Subsequently we obtained the TDDFT linear optical response of the cluster within the so-called adiabatic local density approximation, i.e., using the LDA exchange-correlation kernel. The calculations were performed using an efficient iterative scheme ${ }^{84-86}$ that allows to obtain the optical response at the TDDFT level for large clusters and molecules with moderate computational resources. ${ }^{87}$ The technical details of the method can be found in the Supp. Inf. The results of our atomistic simulations are compared to TDDFT-LDA simulations performed for Na clusters, described within the jellium model (JM). ${ }^{63}$ In the JM only the valence electrons are treated explicitly, and the ionic cores are represented through an homogeneous positive charge density bound by the cluster boundary (jellium edge). In the comparison, we use JM spheres of radius $15.57 \AA$, and fix the averaged electron density to that of bulk Na (Wigner-Seitz radius $\mathrm{r}_{s}=4$ a.u.). The resulting closed-shell JM cluster contains 398 electrons and is similar in size to the $\mathrm{Na}_{380}$ cluster tackled in the atomistic calculations.

We first explore the atomic-scale near-field confinement in a single plasmonic nanoparticle, constituted here by a $\mathrm{Na}_{380}$ cluster. The polarizability of the $\mathrm{Na}_{380}$ cluster obtained with the atomistic simulations shows a well-defined plasmonic peak near $3.35 \mathrm{eV}$, associated with a typical dipolar excitation along the axis of the external driving field. Further technical details of the calculation are addressed in the Supp. Inf.

Figure 1 shows the local near-field distribution around the $\mathrm{Na}_{380}$ cluster for different polarizations of the incident field of amplitude $E_{o}$, both at the dipolar plasmonic resonance energy (panels a-c) and out of resonance (panels d-f). The orientation of the cluster relative to the external field is further clarified in Figure S1 in the Supp. Inf. We show the data in the $(y, z)$ plane passing through the center of the cluster for two different incident linear 
polarizations (along $y$ axis in panel (a) and along $z$ axis in panel (b)). As observed in the plots, when the atomistic structure is accounted for, the near fields dramatically depend on the cluster orientation with respect to the polarization direction. Even if the general dipolar pattern of the induced fields is preserved, the underlying icosahedral geometry of the atomic arrangement can be clearly recognized. Most importantly, we can clearly identify sub-nanometric 'hot-spots' characterized by strongly localized fields at the metal-vacuum interface. The enhancement at these 'hot-spots' is not dramatically larger than that of the overall background of the plasmon-enhanced near fields, but it carries a very distinctive localization with it. The 'hot-spots' of the $\mathrm{Na}_{380}$ cluster in panels (a) and (b) are related to the atomic-scale vertices and edges of the icosahedron cluster structure. Obviously, a quantum (or classical) calculation that considers smooth surfaces and thus ignores the atomistic nature of the particles cannot address these subnanometric features in the near fields, and misses the description of atomic-scale field localization. This is illustrated in panel (c) where the results based on the JM for the perfectly spherical cluster are shown for comparison. In this case, the induced fields are independent of the cluster orientation with respect to the incident field, and feature the typical smooth dipolar pattern of the plasmon induced along the polarization direction ( $y$ axis chosen here). While outside the cluster the TDDFT-JM description is very similar to the classical $\mathrm{Mie}^{88}$ results for a metal sphere described with a Drude dielectric function, quantum effects are apparent inside the cluster where the screening of fields is accompanied by Friedel-like oscillations. ${ }^{89}$

Remarkably, in the atomistic results we observe an atomic-scale lightning rod effect ${ }^{65}$ that can be related to the macroscopic lightning rod effect ${ }^{66,90}$ resulting from the classical field divergence at infinitely sharp tips. The atomistic structure of the material naturally sets a quantum constraint to the notion of "infinitely sharp" since, obviously, an effective curvature radius cannot be smaller than that given by the electron density profile of the single atom. The local dipoles responsible for the subnanometric field confinement are formed by the collective response of the protruding groups of atoms screened by the rest of the 

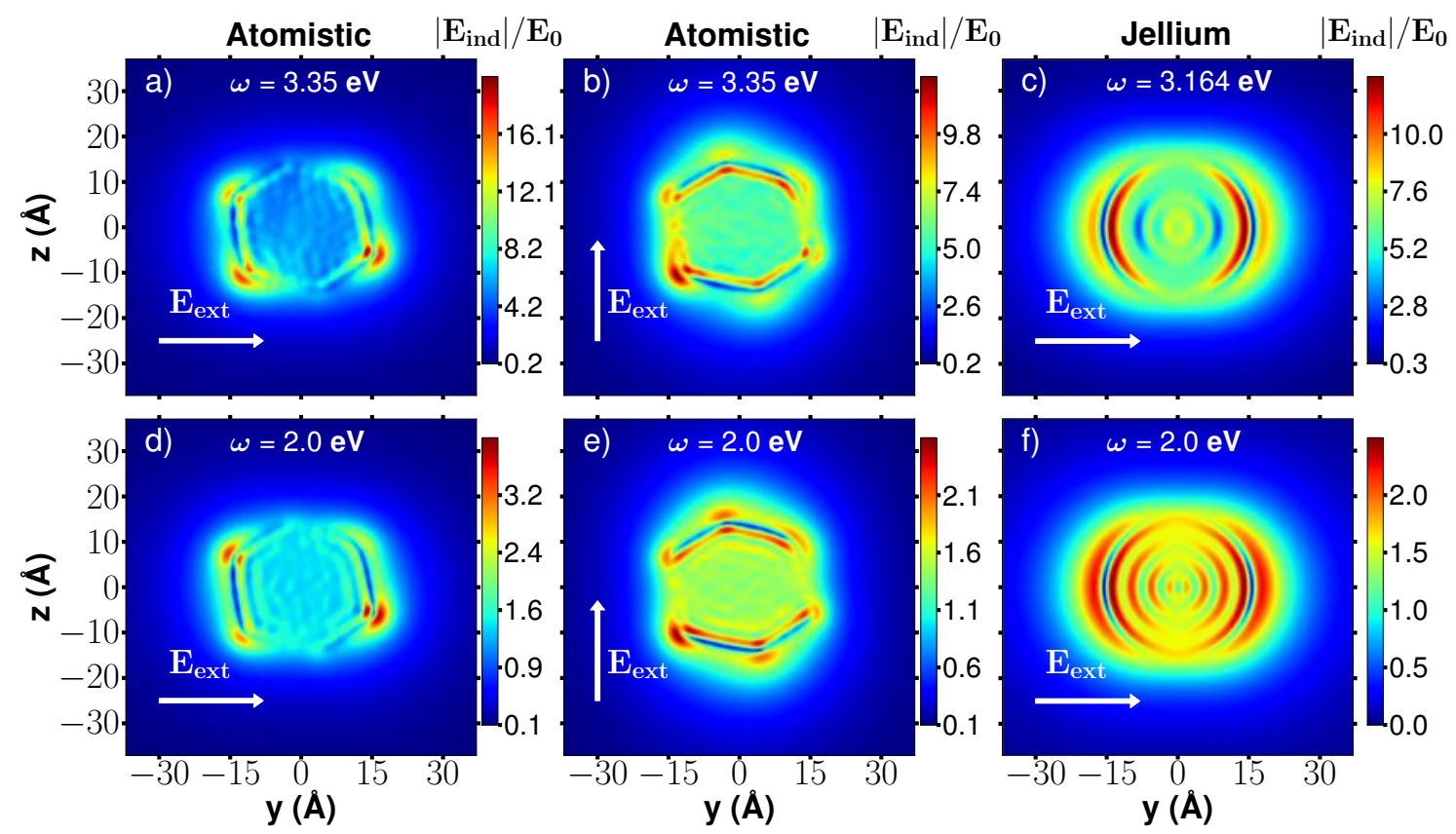

Figure 1: Induced-field enhancement, $\left|\mathbf{E}_{\text {ind }}\right| / \mathrm{E}_{o}$, with $\mathrm{E}_{o}$ the amplitude of the incident field, in the proximity of a $\mathrm{Na}_{380}$ cluster and a jellium sphere $\left(\mathrm{r}_{s}=4\right.$ a.u.) of radius $\mathrm{R}=15.57 \AA$. The induced field is represented in the $(y, z)$ plane, that passes through the center of the cluster/sphere. Top panels show the results at the dipolar plasmonic excitation (at $3.35 \mathrm{eV}$ and $3.16 \mathrm{eV}$, for the atomistic and jellium models, respectively), (a) for a polarization of the external field along the $y$ axis, (b) along the $z$ axis, and (c) along the $y$ axis, (although the response is isotropic in the jellium case). (d)-(f) show the same information as (a)-(c) for an energy of the exciting field out of resonance $(2 \mathrm{eV})$. The orientation of the $\mathrm{Na}_{380}$ particle with respect to the coordinate axis is clarified in Figure S1 in the Supp. Inf. 
cluster. Therefore, at resonance excitation conditions, the near field structure of the cluster, as obtained from our atomistic calculations, can be understood as a combination of two effects: (i) The overall plasmonic near-field enhancement at the dipolar mode, and (ii) The atomic scale lightning rod effect which arises because of the presence of vertices and edges between the atomic planes forming the cluster surface, allowing to further focus the energy into an extremely small area. We can thus establish an analogy with the macroscopic selfsimilar plasmonic nanoantennas, where larger antennas produce further enhancement on the smallest ones, like in a plasmonic lens. ${ }^{91-93}$ Here, the sub-nanometer 'hot-spots' induced around atomically sharp features are fed in a cascade fashion by the plasmonic field of the larger and smoother nanometric plasmonic system (hosting particle or dimer).

The lightning rod effect can be isolated in the absence of a plasmonic resonance. We illustrate this in Fig. 1(d)-(f) where we plot the near-field distribution around the $\mathrm{Na}_{380}$ cluster, obtained for incident plane wave irradiation off resonance with the plasmonic dipolar mode. Except for this change of wavelength, we used the same conditions regarding polarization, geometry, and atomistic or jellium modeling of the cluster, as in panels (a)-(c). In the offresonance situation, the atomic-scale 'hot-spots' are still present for the $\mathrm{Na}_{380}$ cluster for both polarizations, providing a substantial local enhancement that extends a few Ångstroms from the cluster surface. In this case, the lightning rod effect is isolated from the plasmonic effect, even though overall, in the absence of the resonant dipolar plasmon mode, the induced near fields are several times weaker, which also holds for the 'hot-spots' regions.

We emphasize next the importance of atomic-scale features in a canonical structure in plasmonics, the metallic nanogap. By placing two metallic nanoparticles together, we can create a plasmonic nanogap where the coupling of plasmonic modes generates new hybridized solutions that are redshifted with respect to the original resonances of the individual particles. ${ }^{3,94}$ In Fig. 2 we analyse the importance of the atomistic details of the nanogap by selecting results obtained for three main configurations that present different terminations of the interfaces at the gap: (i) facet-to-facet, (ii) tip-to-facet, and (iii) tip-to-tip. For com- 
pleteness, we also consider the case of a dimer formed by two jellium spheres, thus forming a nanogap bound by smooth surfaces. The spectral behavior of the nanogap resonances as a function of the interparticle separation distance, $d_{\text {sep }}$, is displayed in Fig. 2 (a)-(d), where we plot the absorption cross-section, $\sigma_{\mathrm{abs}}$, for each case: (a) facet-to-facet, (b) tip-to-facet, (c) tip-to-tip, and (d) jellium. The polarization of the incident field is set along the dimer axis.

The corresponding atomistic structure of the different nanogap models can be found in Fig. 2 (e)-(g), along with the distribution of the induced near-field for an interparticle distance of $10 \AA$, evaluated at the resonance frequency for each type of gap. In the case of the atomistic calculations, the separation distance, $d_{\text {sep }}$, is measured between the closest atoms across the gap. Separation distances smaller than $1 \AA$, as well as negative distances (corresponding to the case of overlapping clusters $^{3}$ ), have been modeled using modified structures in which atomic layers are successively removed from one of the clusters (areas marked with $\mathrm{A}, \mathrm{B}$ and $\mathrm{C}$ at the bottom of the figures). More information on the actual structures can be found in the Supp. Inf. For a consistent comparison, in the jellium case we also consider the separation distance as defined from the surface atomic layers rather than from the jellium edges.

In Figs. 2(a)-(d) we can distinguish clearly the evolution of the hybridized Bonding Dimer Plasmon (BDP) for each gap configuration: the BDP redshifs as the separation distance between the nanoparticles decreases. ${ }^{3,28,29}$ Consistent with previous descriptions, this trend holds down to separations of the order of a few Angstroms. When the particles are even closer together, the system enters a new regime due to the emergence of the tunneling current across the gap at optical frequencies. ${ }^{62,63,95}$ In the quantum tunneling regime, the BDP is progressively screened, and it disappears from the spectrum. ${ }^{62,63,95}$ For increasing current across the gap, charge-transfer plasmons $(\mathrm{CTPs})^{96}$ that correspond to the polarization of the entire dimer with interparticle charge transfer, emerge. Despite the common general trends for the three atomistic calculations, remarkable differences arise with regard to the 

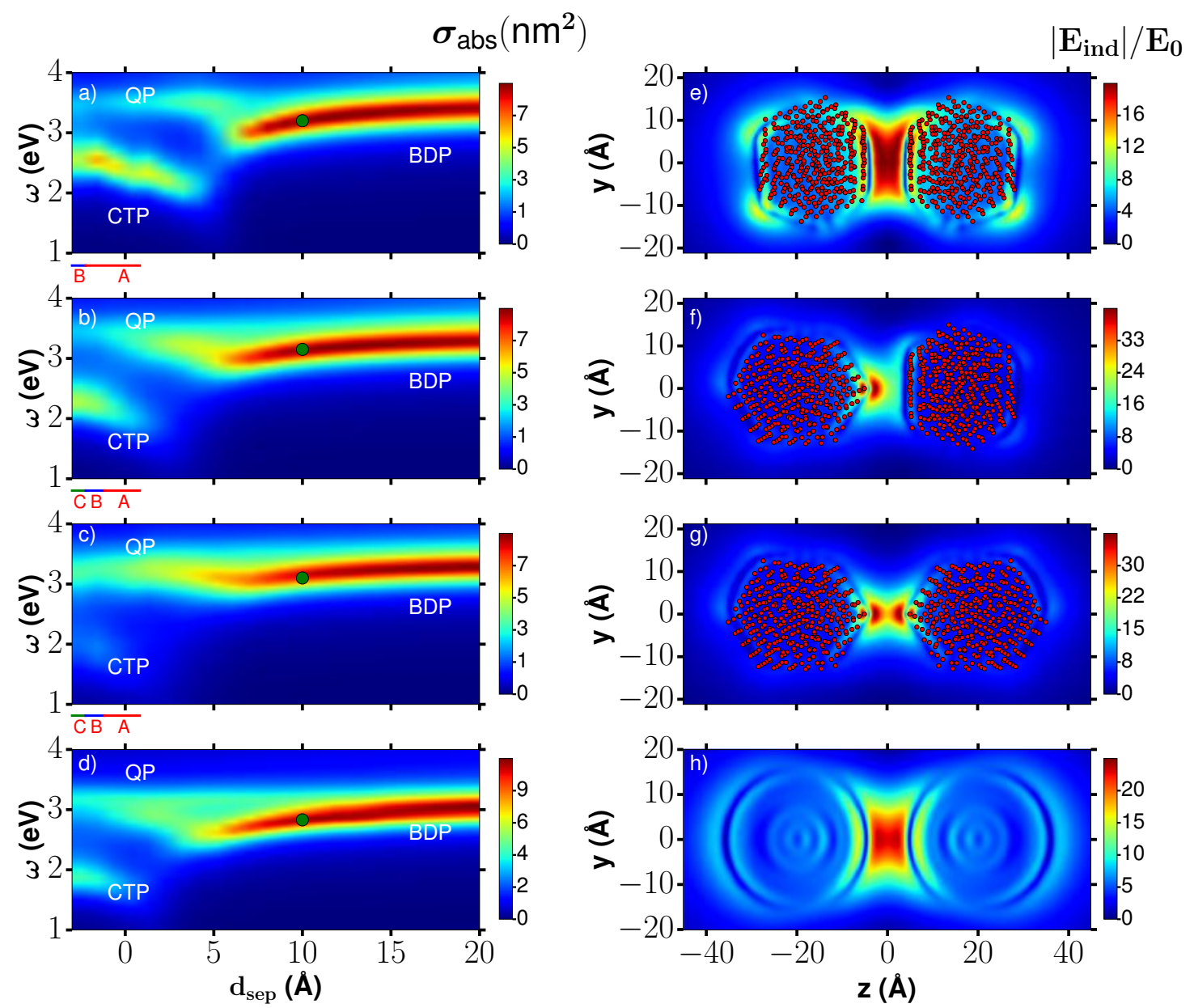

Figure 2: Panels (a)-(d) show the spectral evolution of the absorption cross-section of the plasmonic dimers depicted in (e)-(g), and a dimer of jellium spheres (h), for a polarization of the incident field along the dimer axis as a function of separation distance between the particles, $d_{\text {sep }}$. The hybridized bonding dipolar plasmon (BDP), the charge-transfer plasmon (CTP) mode and the quadrupolar (QP) are identified on the spectra. Separation distances lower than $1 \AA$, and negative distances represent overlapping clusters and have been modeled in (a)-(c) by modified geometries in which atomic layers of one of the clusters are subsequently removed (region A, one layer removed; region B, 2 layers; region C, 3 layers). Panels (e)-(h) show the distribution of the local induced-field produced in a longitudinal cross section $(\mathrm{x}=0$ plane) of the dimer for an energy in resonance with the BDP and for a separation distance of $10 \AA$ : (e) the gap is formed by a facet-to-facet junction, (f) facet-to-tip, and (g) tip-totip configurations. In (h) the same situation for a dimer described by the jellium model is displayed. 
exact separation distance where the quantum effects occur. For the same separation distance $d_{\text {sep }}$, in the facet-to-facet configuration the tunneling current density is larger because of the larger contact area. Thus the BDP disappears and the CTP emerges at the largest separation distances, (6 $\AA$ and $4 \AA$ respectively). In the configurations characterized by the presence of a tip, the tunneling current is confined mainly to the area around the tip, thus the overall tunneling current is smaller. As a consequence, smaller separation distances, $d_{\mathrm{sep}}$, are required for the quenching of the BDP and appearance of the CTPs in such cases, as clearly observed in Figs. 2(b) and (c).

We now explore the influence of the different atomistic configurations of the plasmonic gap on the near-field distribution at the resonance position. In Figs. 2(e)-(h) the induced near-fields are shown in the $(y, z)$ plane of the dimer passing through the centers of the nanoparticles for the facet-to-facet (e), tip-to-facet (f), tip-to-tip (g), and jellium spherical (h) configurations. The width of the gap is set in all the cases to $d_{\text {sep }}=10 \AA$, with incident light in resonance with the hybridized BDP corresponding to the position marked with a green dot on the spectra to the left. The Coulomb coupling between induced charges of opposite signs across the gap leads to a strong localization and enhancement of the near fields in the gap. This effect is widely exploited in surface-enhanced spectroscopies and microscopies. Overall, the nanometric near-field distributions obtained in the full atomistic calculations at the BDP frequency show similar gross features to those in the JM calculations. However, the exact atomistic structure of the junction determines the details of the nearfield distribution, and in particular the appearance of the extremely localized 'hot spots'. As observed in Fig. 2(e), the gap characterized by a facet-to-facet configuration features a welldefined and homogeneous field enhancement that extends over the entire gap, as expected from a scaled parallel plate capacitor. The lateral localization of the hot-spot between the particles is thus determined by the corresponding nanometric facet size. Albeit much less intense, atomic-scale hot spots can be also identified at the edges and vertices of the different facets of the nanocrystal surfaces, both near and opposite to the plasmonic gap. These are 
due to the atomic-scale lightning rod effect, similar to that found in Fig. 1 for the single particle. Compared to the JM with a perfectly spherical geometry, atomic-scale features between the cluster facets increase the Landau damping of the BDP and thus broaden the plasmon resonance peaks (see Supp. Inf.).

A remarkable situation is achieved when exploiting the atomic-scale lightning rod effect in a tip-to-facet or in a tip-to-tip configurations in the gap [Fig. 2(f) and (g)]. In such situations, the tip-induced enhancement is superimposed on the already intense background field of the plasmonic resonance, producing a further increase of the value of the enhancement, and more importantly, an extreme localization of this local near-field down to an extension of a few Ångstroms. This extreme confinement of the fields is missed in the JM model that assumes a smooth density profile, as shown in Fig. 2(h). By means of our realistic atomistic calculations, we have thus shown that atomic-scale hot spots are possible, and they may be relevant to allow superresolution in a variety of experimental near-field techniques. ${ }^{49,70,71}$

To analyze the field enhancement induced at each of the plasmonic cavities quantitatively, we show in Fig. 3 the maximum induced near-field enhancement at resonance in the middle of the gap, $\left|\mathbf{E}_{\text {ind }}^{\max }\right| / \mathrm{E}_{0}$ [panel (a)], and the effective localization area of the near-field, $\mathcal{A}$ [panel (b)], as a function of the gap separation $d_{\text {sep }}$. We have chosen to plot and analyze the field distributions at those resonance energies that produce the largest maximum of the induced near-field at each configuration. Thus, for the tip-to-facet and tip-to-tip gaps we follow the BDP-QP mode around $3 \mathrm{eV}$, whereas for the facet-to-facet configuration data, for $\mathrm{d}_{\text {sep }} \leq 5 \AA$ we follow the CTP mode appearing at lower energies. The local near-fields are obtained for the same configurations and light incidence as in Fig. 2. The maximum enhancement is found in all the cases for $d_{\text {sep }} \approx 7 \AA$ [see Fig. 3(a)]. As discussed in the detail in the Supp. Inf., when the gap size is reduced below this value, the electron tunneling neutralizes the plasmon-induced charges at the metal surfaces across the gap, and reduces the induced fields. ${ }^{62,63}$ For larger separations, the coupling between the plasmons of the individual particles becomes smaller thus the field enhancement progressively decreases, and 
the differences due to the atomic-scale features are also attenuated. When atomic-scale $\operatorname{tip}(\mathrm{s})$ is (are) present in the junction, the maximum near-field enhancement is about 1.5 times larger than that of a facet-to-facet configuration [red and blue lines vs. green line in Fig. 3(a)], emphasizing the importance of the fine details of the gap.

Together with the absolute value of the field enhancement, the confinement is an aspect of particular interest in nanophotonics. In Fig. 3(b), we plot the effective localization area, $\mathcal{A}$, of the BDP for each atomistic configuration. $\mathcal{A}$ is defined according to the following expression:

$$
\mathcal{A}=\frac{1}{h} \int_{V} \frac{\left|\mathbf{E}_{\text {ind }}(x, y, z)\right|^{2}}{\left|\mathbf{E}_{\text {ind }}^{\max }\right|^{2}} d V,
$$

where $\left|\mathbf{E}_{\text {ind }}(x, y, z)\right|$ is the modulus of the induced field in a thin slab of volume $V$ and thickness $h=0.63 \AA$ in the $z$ direction, with $z=0$ at the center of the gap. $\left|\mathbf{E}_{\text {ind }}^{\max }\right|$ is the maximum value of the field in the integration volume. Therefore, $\mathcal{A}$ provides a measure of the effective area in which the induced field is localized within the middle of the gap. The results are plotted in Fig. 3(b) as a function of separation distance, for the same excitation wavelengths used in Fig. 3(a). As expected, the three different atomistic configurations show maximum localization of the BDP mode at separations of about 7 Angstroms, corresponding to the separation of maximal enhancement described above. For smaller separation distances, when the tunneling current is established, the field is expelled out from the gap and thus becomes less localized. ${ }^{49}$ The tip-to-tip morphology, for which the lightning rod effect is more pronounced, provides the strongest localization among all. The minimum $\mathcal{A}$ in this case is $0.4 \mathrm{~nm}^{2}$, clearly indicating that the plasmonic fields can be localized down to lateral dimensions of a few thousandths of the incident wavelength with the help of an atomic feature. This value of $\mathcal{A}$ is up to 4 times smaller than that for the facet-to-facet configuration, for the same $d_{\text {sep }}=7 \AA$.

For reference purposes, we also plot in Fig. 3(b), as a black dashed line, the localization corresponding to a classical calculation of the BDP in a gap formed by spherical particles of the same size, and characterized by a Drude-like response that corresponds to the same 


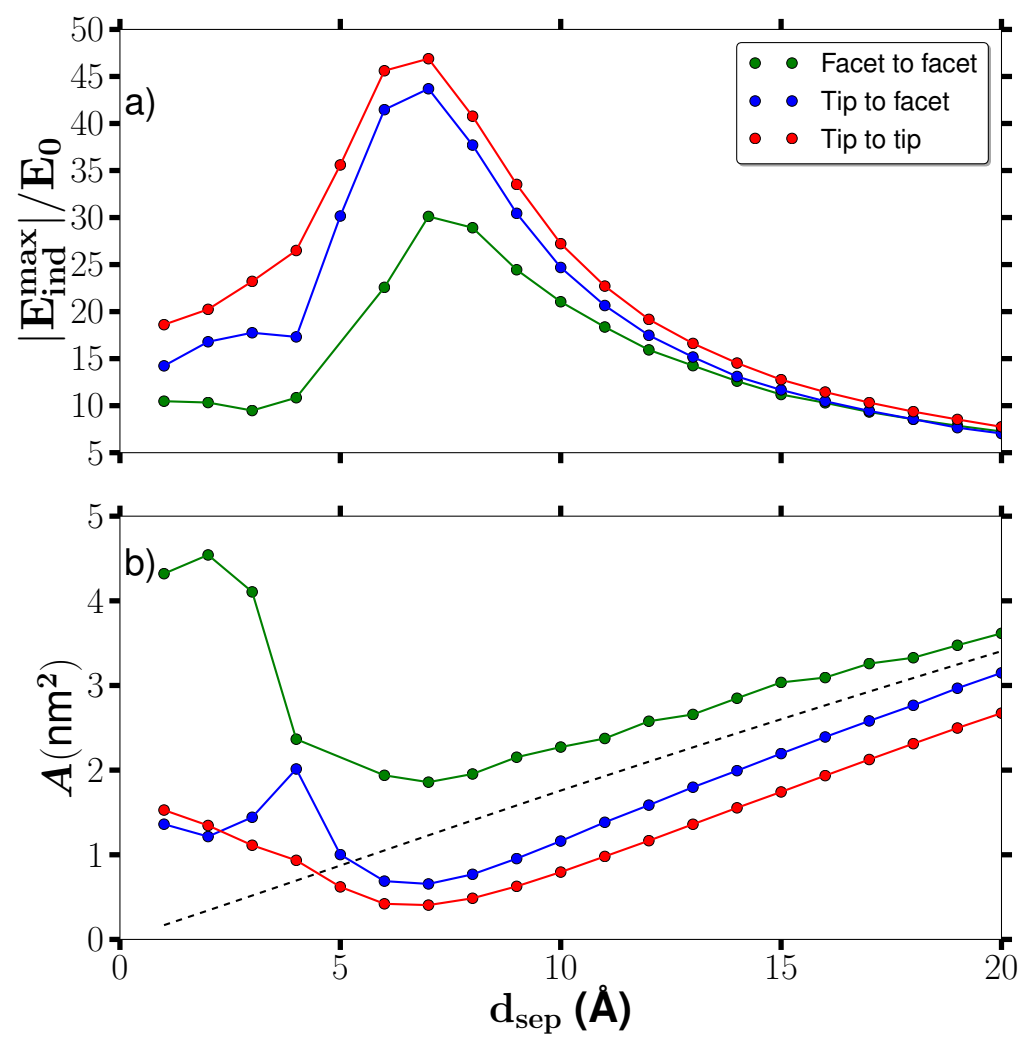

Figure 3: (a) TDDFT calculation of the maximum enhancement of the local induced-field $\left|\mathbf{E}_{\text {ind }}^{\max }\right| / \mathrm{E}_{o}$ at the center of a gap between two particles showing different atomistic configurations, as a function of the separation between the particles forming the gap, $d_{\text {sep }}$. The separation is defined as the distance between the most protruding atoms in both clusters. The green line stands for the facet-to-facet configuration in the gap, the blue line stands for the tip-to-facet configuration and the red line stands for the tip-to-tip configuration. (b) Effective localization area $\mathcal{A}$ of the local field in the midplane of the gap as defined in the text [Eq. (1)] for each of the three configurations. As a reference, the localization of the BDP for a pair of spherical particles given by a classical calculation is displayed as a dashed line. The plotted data correspond to the resonance energies that produce the largest maximum of the induced near-field for each configuration and distance. For the facet-to-facet case this corresponds to the CTP mode for $\mathrm{d}_{\mathrm{sep}}<5 \AA$. For the the tip-to-facet and tip-to-tip gaps we follow the $\mathrm{BDP}$ and $\mathrm{QP}$ modes around $3 \mathrm{eV}$. The different dependence of $\mathcal{A}$ on $d_{\text {sep }}$ for the $\mathrm{BDP}$ and QP modes is clearly visible in the singular behavior of the $\mathcal{A}$ data around $4 \AA$ for the tip-to-facet case, which is almost undetectable in the more gradual BDP-QP transition of the tip-to-tip gap. 
electronic density as in the atomistic calculations. As observed in the figure, the localization in this case tends linearly to zero for small $d_{\text {sep }}$. This unphysical result is due to the lack of dynamical screening and tunneling in the classical description. It is interesting to note that all the systems show a linear dependence of the localization with separation distance, as the gap is opening. This is a reminiscence of the two effects involved: the overall plasmonic effect, plus the atomistic effect. The classical estimation in spherical particles indeed establishes a reference for the behaviour of the localization at the different atomistic gaps: when an atomic-scale tip is present in the gap, the linear dependency is pushed below this classical result (larger slope of red and blue lines). However, for the case of a facet-to-facet gap, the linear dependency shows a smaller slope, setting values of the localization area that exceed those of the classical confinement. In this case the minimal localization area of the field is approximately given by the surface of the facet, as one would expect for a flat capacitor. Therefore, our classical result for the induced-field localization establishes a good benchmark to distinguish between subplasmonic and plasmonic localization, depending on whether the linear tendencies show a more or less pronounced change, respectively, compared to the classical reference of spherical particles.

As a further remark, it should be noted that the presence of subnanometric 'hot spots' would only weakly affect $\mathcal{A}$, as defined by Eq. (1), in large plasmonic systems because the integral will be dominated by the overall plasmonic field structure in the gap. This is actually one of the reasons why plasmonic enhancement is a robust and reliable tool in many standard field-enhanced spectroscopies. Nonetheless, such local behavior at the atomic scale can be important to determine the precise properties of the near field at particular positions, probed, for example, by molecular targets ${ }^{69,72}$ or by electron emission, ${ }^{97}$ which are extremely sensitive to these spatial inhomogeneities independently of how large the plasmonic background is.

The quantitative analysis of the evolution of the near fields with the gap size performed in Fig. 3 is further illustrated in Fig. 4 and Fig. 5 which allow for a more intuitive insight into the role of the atomistic structure on the localization. In Fig. 4 we plot the distribution 
of induced near fields in the $(y, z)$ plane containing the centers of the nanoparticles. The results are shown for the facet-to-facet (left column), tip-to-facet (middle column), and tipto-tip (right column) configurations of the nanogap. Three separations, $d_{\text {sep }}=20 \AA$ (top row), $d_{\text {sep }}=6 \AA$ (middle row), and $d_{\text {sep }}=1 \AA$ (bottom row), are chosen here to represent respectively weak interaction, strong interaction and strong tunneling regimes. For the largest separation, $d_{\text {sep }}=20 \AA$, the coupling between nanoparticles is weak, therefore the near fields around each nanoparticle of the dimer resemble those of the individual particles, as presented in Fig. 1. Nevertheless, stronger fields at the facing surfaces across the gap can be clearly observed, indicating the onset of the hybridization of the BDP.
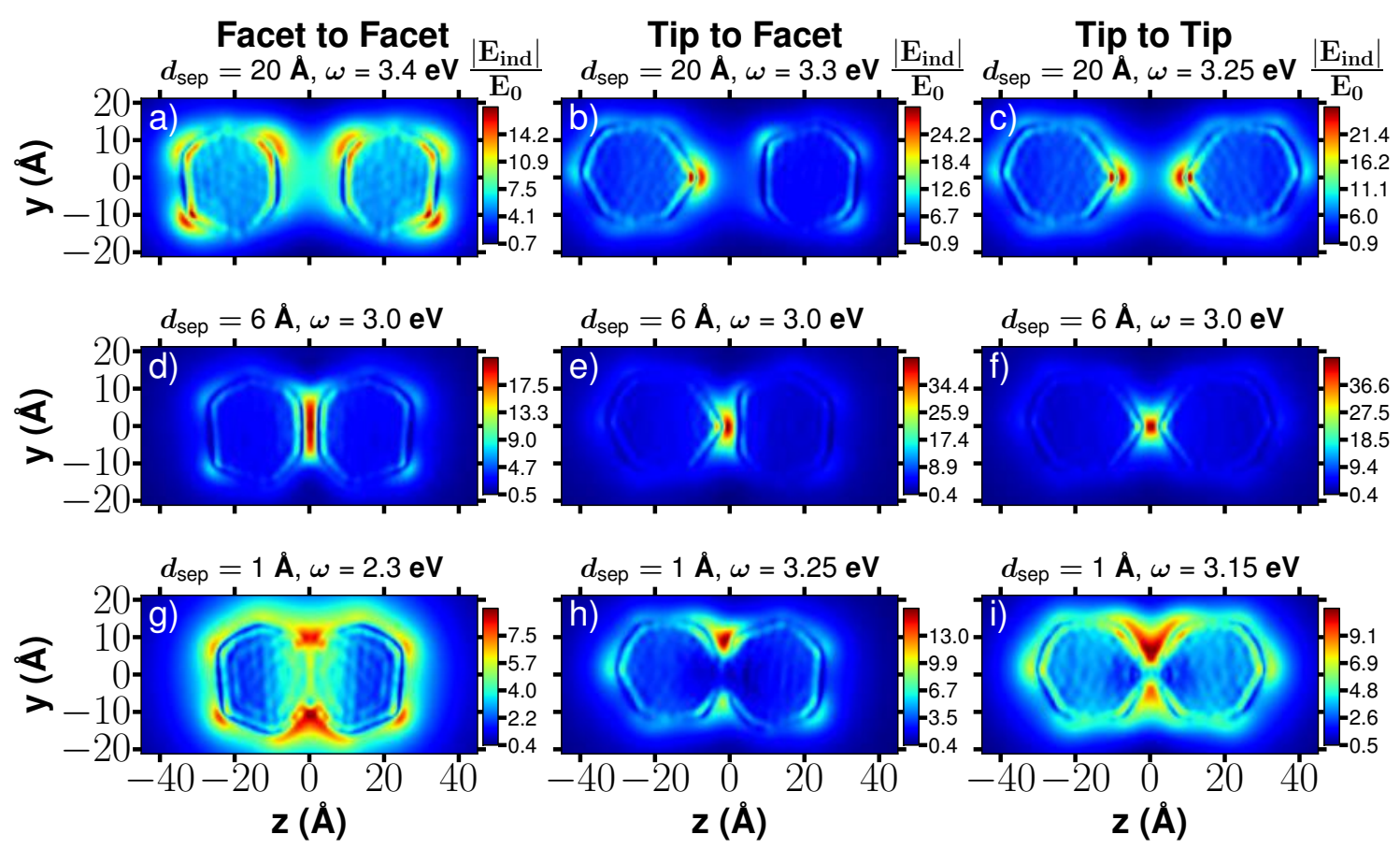

Figure 4: Local induced-field enhancement in a transversal cross section that contains the axis of a $\mathrm{Na}$ metallic dimer for the case of a facet-to-facet configuration at the gap (left column), tip-to-facet configuration (middle column) and tip-to-tip configuration (right column). From top to bottom, three cases of decreasing separation distance are shown for each case: $d_{\text {sep }}=20 \AA$ (top row, a-c), $d_{\text {sep }}=6 \AA$ (middle row, d-f) and $d_{\text {sep }}=1 \AA$ (bottom row, g-i). The field is always plotted at the frequency of the most intense resonance in the absorption cross-section of the dimer. This corresponds to the BDP mode in most cases, except for the facet-to-facet and tip-to-face cases at the closest separation, that corresponds to the CTP mode (panel g and $\mathrm{h}$ ). 
Atomic-scale 'hot-spots' are visible all over the nanoparticle surface, particularly in regions associated with atomic edges at the contact of the crystalline facets. These 'hot-spots' are apparent for the flat-facet gap (top row, left), but are even more pronounced in the presence of a tip-like geometry pointing towards the junction, and aligned with the polarization direction (middle and right). As the particles get closer, $d_{\text {sep }}=6 \AA$ (middle row), the field enhancement at the gap becomes very intense (notice the absolute value of the corresponding scales). For the facet-to-facet situation (middle row, left column) the enhancement is homogeneous inside the gap as a consequence of the flat-surface capacitor geometry. In contrast, when a tip-like configuration is present in the gap, it leads to a particularly strong field enhancement and to clear subnanometer localization at the center of the gap, consistent with the results of Fig. 3. For very small separation distances (bottom row), the electronic densities of the two particles strongly overlap, essentially producing a nanometric neck of continuous electron density. Therefore, the region of enhanced field is expelled to the edges of the junction. ${ }^{95,96}$ This is accompanied with an overall weakening of the fields in the junction region, which eventually become comparable in intensity to the hot spots at other locations of the dimer surfaces.

The evolution of the field confinement in the gap as a function of separation distance, $d_{\text {sep }}$, and the corresponding change of the localization area $\mathcal{A}$ is illustrated in Fig. 5, where the field enhancement is shown in the $(x, y)$ mid-plane between the two particles for each configuration (facet-to-facet on the left column, tip-to-facet on the middle column, and tipto-tip on the right column). When the particles are far away from each other $\left(d_{\text {sep }}=20 \AA\right.$ ), a relatively broad spatial profile of the plasmonic near-field is obtained (top row). This profile is mainly determined by low order multipoles at each nanoparticle so that the features due to the specific atomistic structures of the nanoparticle surfaces are weak. For smaller separation, $d_{\text {sep }}=10 \AA$, the profile of the near fields reflects the atomistic structure of the nanoparticle surfaces across the gap, showing a triangular shape for the facet-to-facet configuration, a round spot for the tip-to-tip configuration, and a round spot on top of a 


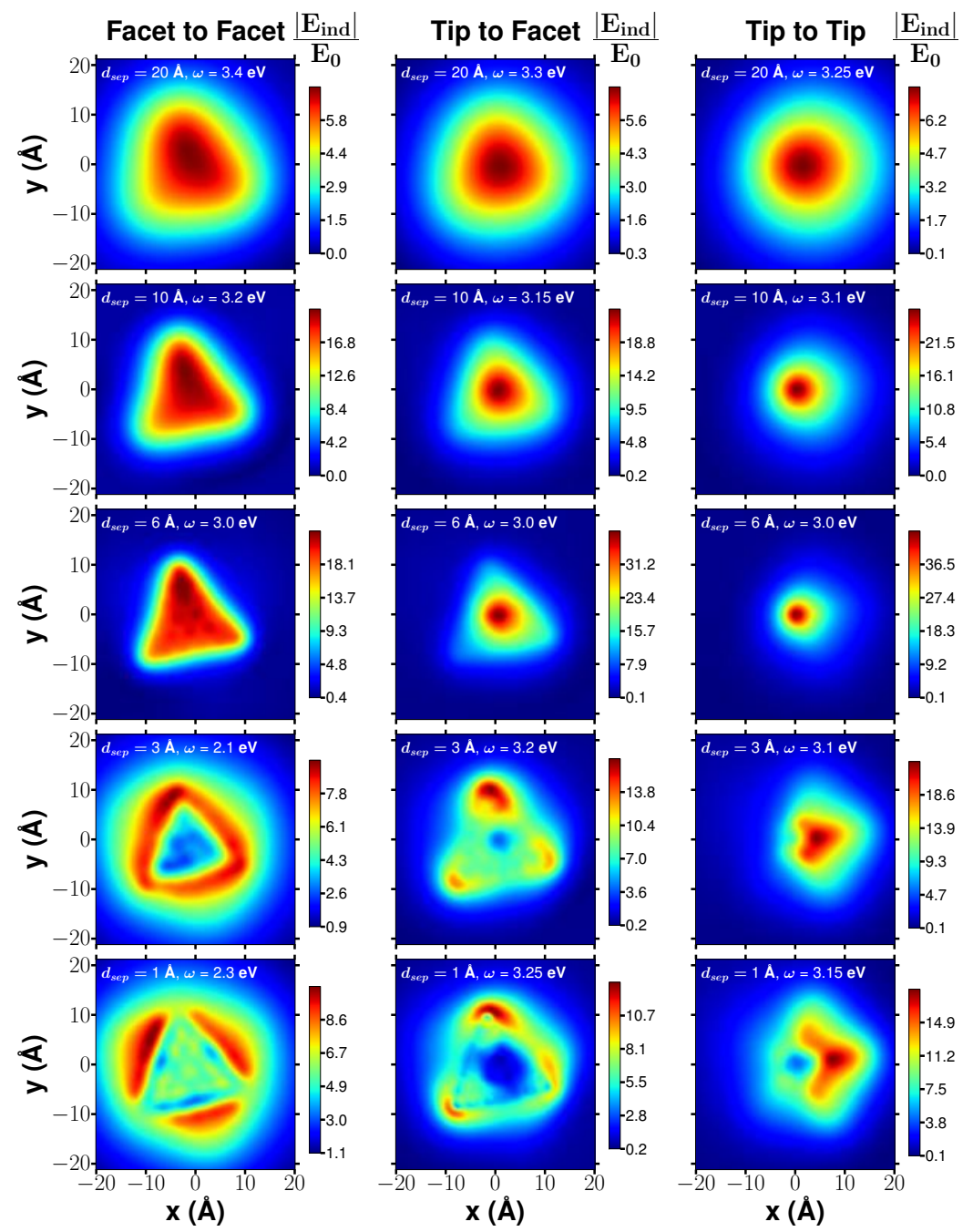

Figure 5: Local induced-field enhancement at resonance in the midplane of the gap between two $\mathrm{Na}_{380}$ clusters for our three configurations, facet-to-facet gap (left column), tip-to-facet (middle column) and tip-to-tip (right column). From top to bottom each case shows a decreasing separation distance for each configuration, from $d_{\text {sep }}=20 \AA$ (largely separated particles, on the top row) to $d_{\text {sep }}=1 \AA$ (interpenetrating situation on the bottom row). The influence of the atomic scale features at the nanogaps is directly noticeable. 
triangular background for the tip-to-facet configuration. As expected from the results in Fig. 3, the tip-to-tip configuration corresponds to the strongest field confinement with the smallest spot size reduced to atomic dimensions, i.e. below $1 \mathrm{~nm}^{2}$ for $d_{\text {sep }} \approx 6 \AA$. For the parallel capacitor facet-to-facet configuration, the spot profile and size change only slightly when $d_{\text {sep }}$ is reduced from $10 \AA$ to $6 \AA$, and the tip-to-facet configuration features the intermediate situation (see also Fig. 4). We thus show here that the widely accepted picture of the overall reduction of the localization area $\mathcal{A}$ for smaller $d_{\text {sep }}$, as obtained from previous studies for smooth particles, ${ }^{62,95,98}$ has to be taken with caution, as it can be altered by the atomistic structure of the gap. For $d_{\text {sep }}$ below $6 \AA$ (lower rows), the tunneling current expels the fields from the middle of the gap. This effect is particularly nicely observed for the CTP mode in the facet-to-facet configuration.

In conclusion, by means of first-principles full-atomistic TDDFT calculations we have demonstrated that the distribution of the near-field close to plasmonic nanoparticles presents subnanometric hot spots that reflect atomic-scale features at the nanoparticle surface. In our case, these features consist of vertices and edges at the contact of the crystallographic facets of the particles. In particular, for the plasmonic dimer, we have shown that the field localization and enhancement inside the plasmonic nanogaps can be very different depending on whether the distribution of the atoms at the gap define a flat surface, or present atomicscale tip-like protrusions. We obtain that the far field also depend on the atomic configuration but in a less marked fashion as expected.

Our findings provide new insights into the limits of plasmonic localization. The presence of atomic-size features, e.g., formed by edges and vertices between crystalline facets in a nanoparticle, gives rise to near-fields localized in regions with linear dimensions of a few Ångstroms, i.e., literally of atomic size. This effect can be related to the classical divergence of a field due to the presence of sharp edges. Indeed, the vertex ending by a single atom, as the one considered here, would be the example of the sharpest possible tip. Furthermore, the field enhanced at the atomic-scale hot spots are intensified by the presence of the overall 
plasmonic background enhancement, following a cascade effect. Based on this parallelism, we can establish an atomic-scale analogy with the macroscopic plasmonic lens of self-similar antennas. ${ }^{91-93,99}$

As demonstrated here, in a realistic nanogap, the key features of the field enhancement can easily reach the atomic-scale. Therefore, a description of the plasmonic response based on smooth interface profiles, either classical or quantum, might not be able to address this atomic-scale near-field regime. To understand and optimize certain experimental situations operating in the subnanometer-scale, it might be mandatory to develop computational schemes that account for the atomistic structural details. Such sensitivity to the atomic details of an structure could explain the lack of reproducibility between apparently similar experiments, ${ }^{100}$ but could also provide a root for further optimization of morphologies. The resolution in optics depends on atomic-scale features at the nanoparticle(s) surface, a remarkable finding that might provide new insights into the limits of plasmon localization, and has important consequences for the limits of optical resolution in field-enhanced spectroscopies ${ }^{101,102}$ and microscopies. ${ }^{49,70,71}$

\section{Notes}

The authors declare no competing financial interest.

\section{Acknowledgement}

We acknowledge useful discussions with Jeremy Baumberg and Volker Deckert regarding practical optical resolution issues in field-enhanced spectroscopy and microscopy, and Dietrich Foerster regarding efficient TDDFT calculations. We acknowledge financial support from projects FIS2013-14481-P and MAT2013-46593-C6-2-P from MINECO. MB, PK, FM and DSP also acknowledge support from the ANR-ORGAVOLT project and the Eurorègion Aquitaine-Euskadi program. MB acknowledges support from the Departamento de Educación of the Basque Government through a PhD grant, as well as from Euskampus and the 
DIPC at the initial stages of this work. RE and PK acknowledge financial support from the Fellows Gipuzkoa program of the Gipuzkoako Foru Aldundia through the FEDER funding scheme of the European Union.

\section{Supporting Information Available}

Supporting Information provides additional details on the computational parameters used our calculations, and how they influence the results, on the structures of the different systems studied here, as well as a more detailed discussion of the data regarding the optical absorption versus $d_{\text {sep }}$ in Fig. 2. This material is available free of charge via the Internet at http: //pubs.acs.org/.

\section{References}

(1) Oldenburg, S. J.; Averitt, R. D.; Westcott, S. L.; Halas, N. J. Chem. Phys. Lett. 1998, $288,243-247$.

(2) Xu, H.; Bjerneld, E. J.; Käll, M.; Börjesson, L. Phys. Rev. Lett. 1999, 83, 4357-4360.

(3) Romero, I.; Aizpurua, J.; Bryant, G. W.; García de Abajo, F. J. Opt. Express 2006, 14, 9988-9999.

(4) Willets, K. A.; Van Duyne, R. P. Annu. Rev. Phys. Chem. 2007, 58, 267-297.

(5) Pelton, M.; Aizpurua, J.; Bryant, G. Laser Photonics Rev. 2008, 2, 136-159.

(6) Alvarez-Puebla, R.; Liz-Marzán, L. M.; García de Abajo, F. J. J. Phys. Chem. Lett. 2010, 1, 2428-2434.

(7) Moskovits, M. Rev. Mod. Phys. 1985, 57, 783-826.

(8) Nie, S.; Emory, S. R. Science 1997, 275, 1102-1106. 
(9) Neubrech, F.; Pucci, A.; Cornelius, T.; Karim, S.; García-Etxarri, A.; Aizpurua, J. Phys. Rev. Lett. 2008, 101, 157403.

(10) Le Ru, E. C.; Etchegoin, P. G. Annu. Rev. Phys. Chem. 2012, 63, 65-87.

(11) Atwater, H. A.; Polman, A. Nat. Mater. 2010, 9, 205-213.

(12) Linic, S.; Christopher, P.; Ingram, D. B. Nat. Mater. 2011, 10, 911-921.

(13) MacDonald, K. F.; Sámson, Z. L.; Stockman, M. I.; Zheludev, N. I. Nat. Photonics 2008, 3, 55-58.

(14) Large, N.; Abb, M.; Aizpurua, J.; Muskens, O. L. Nano Lett. 2010, 10, 1741-1746.

(15) Berrier, A.; Ulbricht, R.; Bonn, M.; Rivas, J. G. Opt. Express 2010, 18, 23226-23235.

(16) Chen, J.; Badioli, M.; Alonso-González, P.; Thongrattanasiri, S.; Huth, F.; Osmond, J.; Spasenović, M.; Centeno, A.; Pesquera, A.; Godignon, P.; Zurutuza Elorza, A.; Camara, N.; García de Abajo, F. J.; Hillenbrand, R.; Koppens, F. H. L. Nature 2012, 487, 77-81.

(17) Lal, S.; Clare, S. E.; Halas, N. J. Acc. Chem. Res. 2008, 41, 1842-1851.

(18) Link, S.; El-Sayed, M. A. J. Phys. Chem. B 1999, 103, 8410-8426.

(19) Jin, R.; Cao, Y.; Mirkin, C. A.; Kelly, K. L.; Schatz, G. C.; Zheng, J. G. Science 2001, 294, 1901-1903.

(20) Averitt, R.; Sarkar, D.; Halas, N. Phys. Rev. Lett. 1997, 78, 4217-4220.

(21) Aizpurua, J.; Hanarp, P.; Sutherland, D.; Kall, M.; Bryant, G. W.; García de Abajo, F. J. Phys. Rev. Lett. 2003, 90, 057401.

(22) Aizpurua, J.; Bryant, G. W.; Richter, L. J.; García de Abajo, F. J.; Kelley, B. K.; Mallouk, T. Phys. Rev. B 2005, 71, 235420. 
(23) Novotny, L. Phys. Rev. Lett. 2007, 98, 266802.

(24) Dorfmüller, J.; Vogelgesang, R.; Weitz, R. T.; Rockstuhl, C.; Etrich, C.; Pertsch, T.; Lederer, F.; Kern, K. Nano Lett. 2009, 9, 2372-2377.

(25) Funston, A. M.; Novo, C.; Davis, T. J.; Mulvaney, P. Nano Lett. 2009, 9, 1651-1658.

(26) Hao, F.; Nehl, C. L.; Hafner, J. H.; Nordlander, P. Nano Lett. 2007, 7, 729-732.

(27) Novikov, S. M.; Sánchez-Iglesias, A.; Schmidt, M. K.; Chuvilin, A.; Aizpurua, J.; Grzelczak, M.; Liz-Marzán, L. M. Part. Part. Syst. Charact. 2014, 31, 77-80.

(28) Su, K.-H.; Wei, Q.-H.; Zhang, X.; Mock, J. J.; Smith, D. R.; Schultz, S. Nano Lett. 2003, 3, 1087-1090.

(29) Rechberger, W.; Hohenau, A.; Leitner, A.; Krenn, J. R.; Lamprecht, B.; Aussenegg, F. R. Opt. Commun. 2003, 220, 137 - 141.

(30) Fan, J. A.; Wu, C.; Bao, K.; Bao, J.; Bardhan, R.; Halas, N. J.; Manoharan, V. N.; Nordlander, P.; Shvets, G.; Capasso, F. Science 2010, 328, 1135-1138.

(31) Hentschel, M.; Saliba, M.; Vogelgesang, R.; Giessen, H.; Alivisatos, A. P.; Liu, N. Nano Lett. 2010, 10, 2721-2726.

(32) Kelly, K. L.; Coronado, E.; Zhao, L. L.; Schatz, G. C. J. Phys. Chem. B 2003, 107, 668-677.

(33) Schnell, M.; García-Etxarri, A.; Huber, A. J.; Crozier, K.; Aizpurua, J.; Hillenbrand, R. Nat. Photonics 2009, 3, 287-291.

(34) Halas, N. J.; Lal, S.; Chang, W.-S.; Link, S.; Nordlander, P. Chem. Rev. 2011, 111, 3913-3961.

(35) Mühlschlegel, P.; Eisler, H.-J.; Martin, O. J. F.; Hecht, B.; Pohl, D. W. Science 2005, 308, 1607-1609. 
(36) Novotny, L.; van Hulst, N. Nat. Photonics 2011, 5, 83-90.

(37) Kern, J.; Großmann, S.; Tarakina, N. V.; Häckel, T.; Emmerling, M.; Kamp, M.; Huang, J.-S.; Biagioni, P.; Prangsma, J. C.; Hecht, B. Nano Lett. 2012, 12, 55045509 .

(38) Ward, D. R.; Hüser, F.; Pauly, F.; Cuevas, J. C.; Natelson, D. Nat. Nanotechnol. 2010, 5, 732-736.

(39) Sigle, D. O.; Mertens, J.; Herrmann, L. O.; Bowman, R. W.; Ithurria, S.; Dubertret, B.; Shi, Y.; Yang, H. Y.; Tserkezis, C.; Aizpurua, J.; Baumberg, J. J. ACS Nano 2015, 9, 825-830.

(40) Danckwerts, M.; Novotny, L. Phys. Rev. Lett. 2007, 98, 026104.

(41) Grillet, N.; Manchon, D.; Bertorelle, F.; Bonnet, C.; Broyer, M.; Cottancin, E.; Lermé, J.; Hillenkamp, M.; Pellarin, M. ACS Nano 2011, 5, 9450-9462.

(42) Sun, M.; Xu, H. Small 2012, 8, 2777-2786.

(43) Christopher, P.; Xin, H.; Marimuthu, A.; Linic, S. Nat. Mater. 2012, 11, 1044-1050.

(44) Mukherjee, S.; Libisch, F.; Large, N.; Neumann, O.; Brown, L. V.; Cheng, J.; Lassiter, J. B.; Carter, E. A.; Nordlander, P.; Halas, N. J. Nano Lett. 2013, 13, 240-247.

(45) Baffou, G.; Quidant, R. Chem. Soc. Rev. 2014, 43, 3898-3907.

(46) Kneipp, K.; Wang, Y.; Kneipp, H.; Perelman, L.; Itzkan, I.; Dasari, R.; Feld, M. Phys. Rev. Lett. 1997, 78, 1667-1670.

(47) Kühn, S.; Håkanson, U.; Rogobete, L.; Sandoghdar, V. Phys. Rev. Lett. 2006, 97, 017402 .

(48) Anger, P.; Bharadwaj, P.; Novotny, L. Phys. Rev. Lett. 2006, 96, 113002. 
(49) Zhang, R.; Zhang, Y.; Dong, Z. C.; Jiang, S.; Zhang, C.; Chen, L. G.; Zhang, L.; Liao, Y.; Aizpurua, J.; Luo, Y.; Yang, J. L.; Hou, J. G. Nature 2013, 498, 82-86.

(50) Ozbay, E. Science 2006, 311, 189-193.

(51) Huang, J.-S.; Feichtner, T.; Biagioni, P.; Hecht, B. Nano Lett. 2009, 9, 1897-1902.

(52) Prangsma, J. C.; Kern, J.; Knapp, A. G.; Grossmann, S.; Emmerling, M.; Kamp, M.; Hecht, B. Nano Lett. 2012, 12, 3915-3919.

(53) Stolz, A.; Berthelot, J.; Mennemanteuil, M.-M.; Colas des Francs, G.; Markey, L.; Meunier, V.; Bouhelier, A. Nano. Lett. 2014, 14, 2330-2338.

(54) Runge, E.; Grosss, E. K. U. Phys. Rev. Lett. 1984, 52, 997-1000.

(55) Parr, R. G.; Weitao, Y. Density-functional theory of atoms and molecules; Oxford University Press: Oxford, 1989.

(56) Feibelman, P. J. Phys. Rev. B 1975, 12, 1319-1336.

(57) Liebsch, A.; Schaich, W. L. Phys. Rev. B 1995, 52, 14219-14234.

(58) Teperik, T. V.; Nordlander, P.; Aizpurua, J.; Borisov, A. G. Phys. Rev. Lett. 2013, 110, 263901.

(59) Monreal, R. C.; Antosiewicz, T. J.; Apell, S. New J. Phys. 2013, 15, 083044.

(60) Negre, C. F. A.; Perassi, E. M.; Coronado, E. A.; Sánchez, C. G. J. Phys.: Condens. Matter 2013, 25, 125304.

(61) Iida, K.; Noda, M.; Ishimura, K.; Nobusada, K. J. Phys. Chem. A 2014, 118, 1131711322.

(62) Zuloaga, J.; Prodan, E.; Nordlander, P. Nano Lett. 2009, 9, 887-891. 
(63) Marinica, D. C.; Kazansky, A. K.; Nordlander, P.; Aizpurua, J.; Borisov, A. G. Nano Lett. 2012, 12, 1333-1339.

(64) Zhang, P.; Feist, J.; Rubio, A.; García-González, P.; García-Vidal, F. J. Phys. Rev. B 2014, 90, 161407.

(65) Gersten, J.; Nitzan, A. J. Chem. Phys. 1980, 73, 3023-3037.

(66) Novotny, L.; Bian, R.; Xie, X. Phys. Rev. Lett. 1997, 79, 645-648.

(67) Le, F.; Brandl, D. W.; Urzhumov, Y. A.; Wang, H.; Kundu, J.; Halas, N. J.; Aizpurua, J.; Nordlander, P. ACS Nano 2008, 2, 707-718.

(68) Zuloaga, J.; Prodan, E.; Nordlander, P. ACS Nano 2010, 4, 5269-5276.

(69) Payton, J. L.; Morton, S. M.; E., M. J.; Jensen, L. J. Chem. Phys. 2012, 136, 214103.

(70) Yano, T.-a.; Ichimura, T.; Kuwahara, S.; HDhili, F.; Uetsuki, K.; Okuno, Y.; Verma, P.; Kawata, S. Nat. Commun. 2013, 4, 2592.

(71) Deckert-Gaudig, T.; Deckert, V. Small 2009, 16, 432-436.

(72) Payton, J. L.; Morton, S. M.; E., M. J.; Jensen, L. Acc. Chem. Res. 2014, 47, 88-99.

(73) Vang, R. T.; Honkala, K.; Dhal, S.; Vestergaard, E. K.; Schandt, J.; Laegsgaard, E.; Clausen, B. S.; Norskov, J. K.; Besenbacher, F. Nature 2005, 4, 160-162.

(74) Woodruff, D. P., Ed. Atomic clusters: From Gas Phase to Deposited; The Chemical Physics of Solid Surfaces, 12; Elsevier Science, Amsterdam, 2007.

(75) Heiz, U., Landman, U., Eds. Nanocatalysis; NanoScience and Technology; Springer Berlin Heildelberg New York, 2007.

(76) Jang, T.; Mowbray, D. J.; Dobrin, S.; Falsing, H.; Hvolbaek, B.; Bligaard, T.; Norskov, J. K. J. Phys. Chem. C 2009, 113, 10548-10553. 
(77) Murrell, J. N.; Mottram, R. E. Mol. Phys. 1990, 69, 571-585.

(78) Noya, E. G.; Doye, J. P. K.; Wales, D. J.; Aguado, A. Eur. Phys. J. D 2007, 43, $57-60$.

(79) The initial structure of the $\mathrm{Na}_{380}$ cluster was download from the Cambridge Cluster Database, D. J. Wales, J. P. K. Doye, A. Dullweber, M. P. Hodges, F. Y. Naumkin, F. Calvo, J. Hernández-Rojas and T. F. Middleton, URL http://wwwwales.ch.cam.ac.uk/CCD.html.

(80) Sánchez-Portal, D.; ; Ordejón, P.; Artacho, E.; Soler, J. M. Int. J. Quantum Chem. $\mathbf{1 9 9 7 ,} 65,453-461$.

(81) Soler, J. M.; Artacho, E.; Gale, J. D.; García, A.; Junquera, J.; Ordejón, P.; SánchezPortal, D. J. Phys.: Condens. Matter 2002, 14, 2745-2779.

(82) Kohn, W.; Sham, L. J. Phys. Rev. 1965, 140, A1133-A1138.

(83) Cohen, M. L.; Dacorogna, M. M. Phys. Rev. B 1986, 34, 4996-5002.

(84) Koval, P.; Foerster, D.; Coulaud, O. J. Chem. Theory Comput. 2010, 6, 2654-2668.

(85) Koval, P.; Foerster, D.; Coulaud, O. Phys. Status Solidi B 2010, 247, 1841-1848.

(86) Foerster, D.; Koval, P. J. Chem. Phys. 2009, 131, 044103.

(87) Manjavacas, A.; Marchesin, F.; Thongrattanasiri, S.; Koval, P.; Nordlander, P.; Sánchez-Portal, D.; García de Abajo, F. J. ACS Nano 2013, 7, 3635-3643.

(88) Mie, G. Ann. Phys.(Leipzig) 1908, 25, 377-445.

(89) Friedel, J. Philos. Mag. 1952, 43, 153-189.

(90) Martin, O. J. F.; Girard, C. App. Phys. Lett. 1997, 70, 705-707.

(91) Li, K.; Stockman, M. I.; Bergman, D. J. Phys. Rev. Lett. 2003, 91, 227402. 
(92) VKravets, V. G.; Zoriniants, G.; Burrows, C. P.; Schedin, F.; Casiraghi, C.; Klar, P.; Geim, A. K.; Barnes, W. L.; Grigorenko, A. N. Phys. Rev. Lett. 2010, 105, 246806.

(93) Höpener, C.; Lapin, Z. J.; Bharadwaj, P.; Novotny, L. Phys. Rev. Lett. 2012, 109, 017402.

(94) Nordlander, P.; Oubre, C.; Prodan, E.; Li, K.; Stockman, M. I. Nano Lett. 2004, 4, 899-903.

(95) Esteban, R.; Borisov, A. G.; Nordlander, P.; Aizpurua, J. Nat. Commun. 2012, 3, 825.

(96) Pérez-González, O.; Zabala, N.; Borisov, A. G.; Halas, N. J.; Nordlander, P.; Aizpurua, J. Nano Lett. 2010, 10, 3090-3095.

(97) García de Abajo, F. J. Rev. Mod. Phys. 2010, 82, 209-275.

(98) Savage, K. J.; Hawkeye, M. M.; Esteban, R.; Borisov, A. G.; Aizpurua, J.; Baumberg, J. J. Nature 2012, 491, 574-577.

(99) Ding, B.; Deng, Z.; Yan, H.; Cabrini, S.; Zuckermann, R. N.; Bokor, J. J. Am. Chem. Soc. 2010, 132, 3248-3249.

(100) Pettinger, B.; Schambach, P.; Villagómez, C. J.; Scott, N. Annual Review of Physical Chemistry 2012, 63, 379-399.

(101) Hartschuh, A.; Sánchez, E.; Xie, X.; Novotny, L. Phys. Rev. Lett. 2003, 90, 095503.

(102) Gerton, J. M.; Wade, L. A.; Lessard, G. A.; Ma, Z.; Quake, S. R. Phys. Rev. Lett. 2004, 93, 180801. 
Graphical TOC Entry

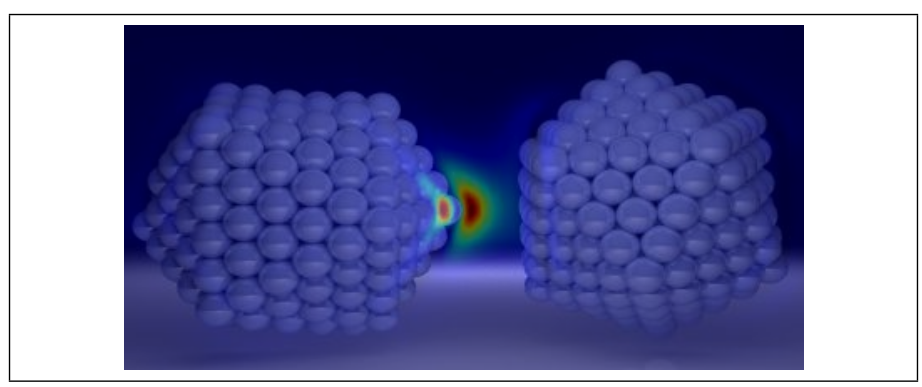




\title{
Supporting Information
}

\section{Atomistic near-field nanoplasmonics: reaching atomic-scale resolution in nanooptics}

\author{
M. Barbry, ${ }^{\dagger}$ P. Koval, ${ }^{\dagger}$ F. Marchesin, ${ }^{\dagger}$ R. Esteban, ${ }^{\dagger}$ A. G. Borisov, ${ }^{\ddagger}$ J. \\ Aizpurua, ${ }^{*, \dagger}$ and D. Sánchez-Portal*, ${ }^{*}$ \\ $\dagger$ Centro de Física de Materiales, Centro Mixto CSIC-UPV/EHU and Donostia \\ International Physics Center (DIPC), 20018 Donostia-San Sebastián, Spain \\ $\ddagger$ Institut des Sciences Moléculaires d'Orsay ISMO, UMR 8214 CNRS-Université \\ Paris-Sud, Bât. 351, Université Paris-Sud, 91405 Orsay Cedex, France
}

E-mail: aizpurua@ehu.es; sqbsapod@ehu.es

\section{Details of the ground-state calculations using SIESTA}

The electronic structure calculations for the $\mathrm{Na}_{380}$ cluster and the $\mathrm{Na}_{380}$ dimers were performed using standard density functional theory (DFT) as implemented in the SIESTA code. $^{1,2}$ The resulting Kohn-Sham orbitals and energies were used as an input for the TDDFT calculations of the optical response using the iterative scheme described in Ref. 3.

We used the local density approximation ${ }^{4,5}$ (LDA), norm-conserving pseudopotentials ${ }^{6}$ to effectively describe core electrons, and a double- $\zeta$ polarized (DZP) basis set of numerical atomic orbitals generated using an energy shift of $100 \mathrm{meV} .{ }^{7}$ The fineness of the real-space grid used to compute the Hartree and exchange-correlation contributions to the energy and Hamiltonian corresponds to a plane-wave cut-off of $150 \mathrm{Ry} .^{2}$ 


\section{Structure of the $\mathrm{Na}_{380}$ cluster and the plasmonic gaps}

Figure $\mathrm{S} 1$ a) shows the relaxed structure of the $\mathrm{Na}_{380}$ cluster whose plasmonic response has been studied in the present paper. The cluster presents an icosahedral structure and has been obtained starting from a configuration optimized with empirical potentials ${ }^{8}$ and available at the Cambridge Cluster Database. ${ }^{9}$ The relaxations were performed using the SIESTA code until forces acting on the atoms were smaller than $0.03 \mathrm{eV} / \AA$.

The structure in Fig. S1 a) is very similar to the initial one, although has a higher density in accordance to the well-known underestimation of the Na lattice parameter by LDA. ${ }^{10}$ This relaxation confirms that the selected structure is stable and at least corresponds to a local minimum of the DFT energy landscape of $\mathrm{Na}_{380}$.

We have highlighted the apex atom of the tip and the facet used to define the different plasmonic nanogaps in our work (see top panel of Fig. S2). The tip has approximately a five-fold symmetry (being formed by the convergence of five facets of approximately similar shape and size). The planar facet (formed by 21 atoms) is an almost equilateral triangle with $\sim 1.5 \mathrm{~nm}^{2}$ area. Panels b), c) and d) clarify the orientation of the isolated cluster used to compute the induced-field distributions in Fig. 1 in the main text of the letter. In those plots the external field is applied along the $y$ or $z$ axes, and the field distribution is plotted in the $x=0$ plane, passing through the center of the cluster and our "tip atom".

As shown in Fig. S2, the plasmonic gaps are formed by two identical $\mathrm{Na}_{380}$ clusters differently oriented. One of the nanogaps (facet-to-facet) is formed by two parallel planar facets, another one is formed by a planar facet and a tip (tip-to-facet), and in the third one two tips face each other (tip-to-tip). The distance between the clusters $\left(d_{\text {sep }}\right)$ is changed and the optical response of the system is monitored as a function of this change in Fig. 2 of the main text. $d_{\text {sep }}$ is defined as the minimal distance between atoms located in different clusters. For $d_{\text {sep }}>1 \AA$ the structures of the clusters (initially relaxed as explained above) remain fixed during the process since the focus of our study is on the modification of the optical response and local field as a function of $d_{\text {sep }}$ for three different, well defined geometries. Clear 

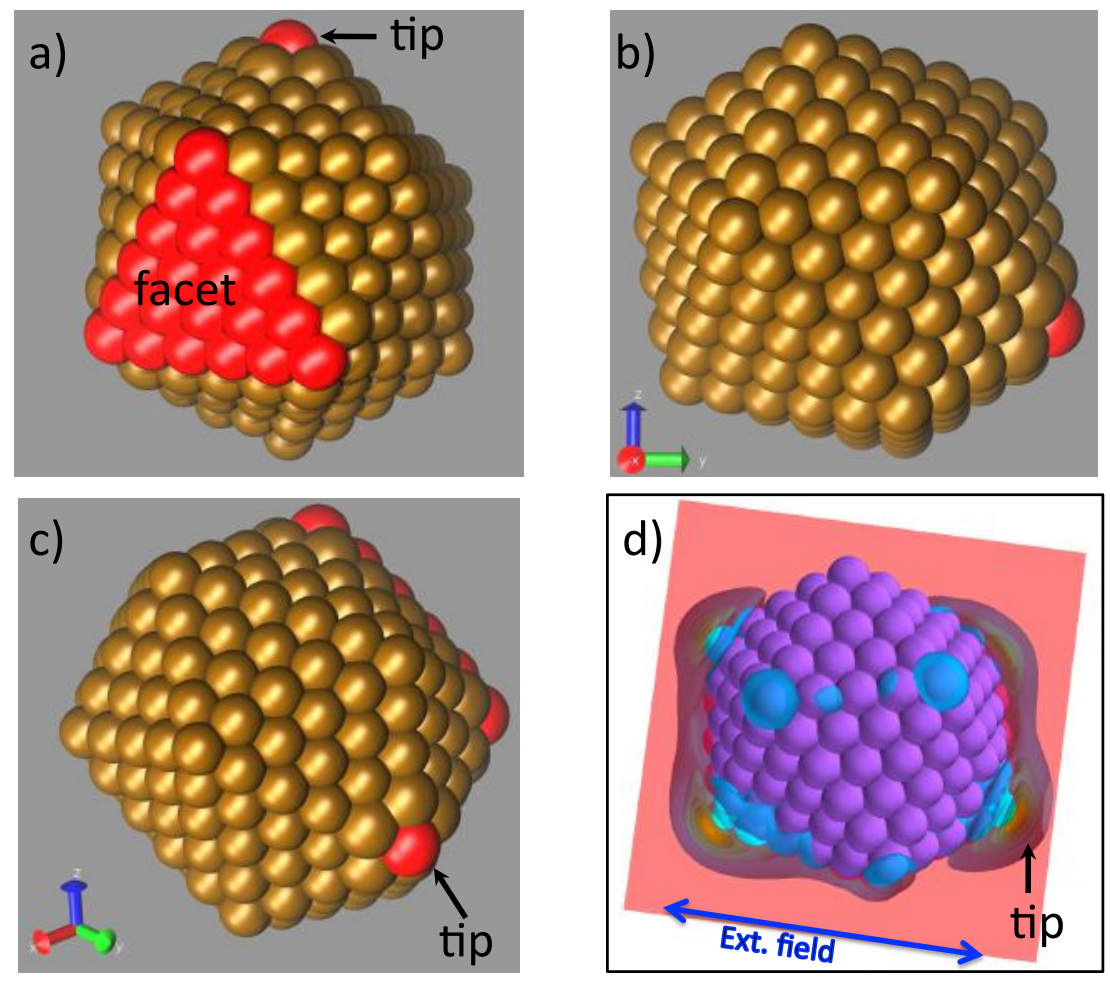

Figure S1: Panel a) shows the atomistic structure of the $\mathrm{Na}_{380}$ cluster after relaxation. The facet and the tip atoms used to define plasmonic gaps of different structures in our calculations have been highlighted in red. Panels b) and c) clarify the orientation, with respect to the coordinate axes, of the cluster used in the calculations of the induced-field distribution of the isolated cluster in Fig. 1 of the main text. Panel d) shows the induced field distribution in a plane passing through the cluster center and containing the "tip atom" $(x=0$ plane) when the external field is applied along the $y$-axis (as used in Fig. 1 of the main text).

shifts of the plasmon resonance and modifications in the distribution and localization of the local fields are found and discussed at length in our paper.

Separation distances $d_{\text {sep }}$ smaller than $1 \AA$ and negative distances represent overlapping clusters, that have been extensively studied using classical electromagnetic simulations. ${ }^{11}$ We have modeled such situations using modified geometries in which atomic layers of one of the clusters are subsequently removed. These structures can be found in panels A, B and C in Fig. S2. In structures type A we remove one layer of atoms (those highlighted in red in the top panels of Fig. S2). In structures of type B we remove a second layer from the same cluster (blue atoms), while configurations of type C (only studied for "tip-cases") are formed 
Facet to facet
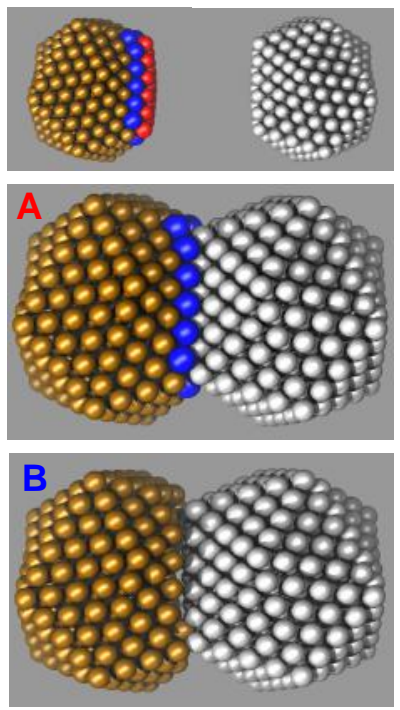

Tip to facet
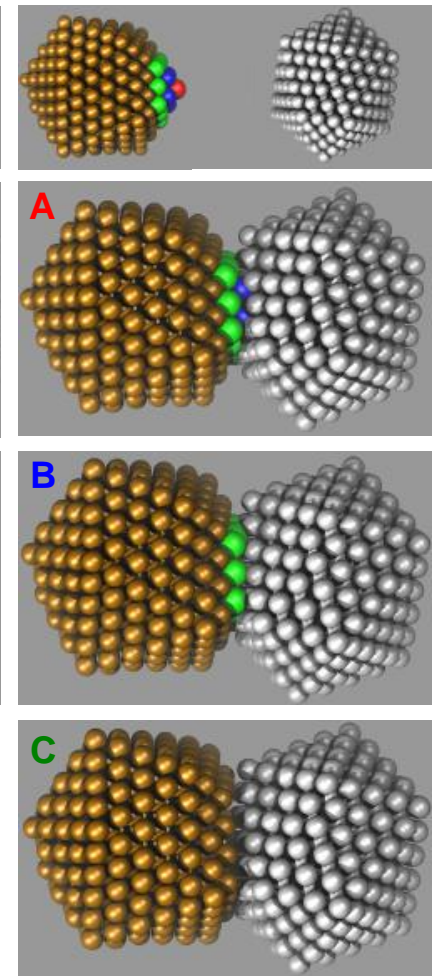

Tip to tip
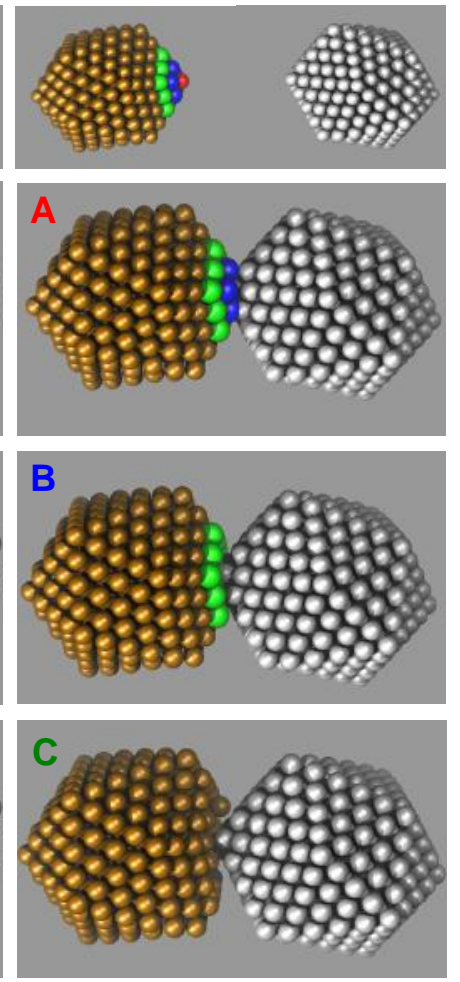

Figure S2: Geometries of the three different $\mathrm{Na}_{380}$ dimers studied in our letter. The top panels show the atomistic structure of the three plasmonic nanogaps for selected inter-cluster distances $\left(d_{\mathrm{sep}}\right)$. The letters in the other panels, and the color code of the atomic layers, refer to those used in Fig. 2 of the main text to label the geometries for $d_{\text {sep }}$ below $1 \AA$, i.e, configurations where the clusters overlap (see the text for a more detailed explanation). These situations are studied to compare with the classical results presented, for example, in Ref. 11.

by removing a third layer (green atoms) in one of the clusters. Remarkably the plasmon resonances and the intensity of the optical absorption changes quite smoothly as we move from configurations type-A, to type-B and C (see Fig. 2 of the main text and Fig. S5 below). 


\section{Details of the TDDFT calculations of $\mathrm{Na}_{380}$ cluster and $\mathrm{Na}_{380}$ dimers}

The calculations of the optical response of sodium clusters and cluster-dimers were performed using time-dependent density functional theory (TDDFT) within the so-called adiabatic local-density approximation, i.e., using the $\mathrm{LDA}^{4,5}$ exchange-correlation kernel computed for the instantaneous electron density. TDDFT is a rigorous (in principle) extension of DFT to time dependent systems. ${ }^{12}$

The ability of the adiabatic time-dependent local density approximation (A-TDLDA) to provide accurate results for the optical properties of small sodium clusters has been demonstrated in several references. ${ }^{13-16}$ For example, Vasiliev et al. ${ }^{14,15}$ have shown by solving Casida equations in a real-space grid that A-TDLDA in conjunction with ab initio pseudopotentials correctly reproduces the shape and peak positions (within 0.1-0.2 eV) of the experimental optical spectrum of very small sodium clusters containing up to 8 atoms. The very good performance of A-TDLDA in the case of the $\mathrm{Na}_{8}$ cluster was also verified by Rubio et $a l .{ }^{13}$ who, using plane-waves and pseudopotentials, obtained a main resonance peak at $2.55 \mathrm{eV}$ in excellent agreement with the experimental value of $2.53 \mathrm{eV}$. Furthermore, the A-TDLDA results for small sodium clusters are also in good agreement with configuration interaction (CI) calculations were core electrons are replaced by effective core potentials. ${ }^{17,18}$

The performance of the A-TDLDA for small sodium clusters has been favorably compared against other more sophisticated functionals and even Bethe-Salpeter equation (BSE) results (see A. Marini, R. Del Sole and A. Rubio in Ref. 19). The authors of these reference compared A-TDLDA results for $\mathrm{Na}_{4}$ against results obtained using the exact-exchange kernel and the GW-BSE approximation. The results are very similar and these authors explicitly concluded that "The LDA kernel is a good approximation of the xc-kernel of small sodium clusters". Since correlation effects are expected to be more prominent for small systems, the fact that clusters formed by as few as $8 \mathrm{Na}$ atoms can be accurately described within A-TDLDA (not 
only matching the experimental results, but also in good agreement with more sophisticated theoretical treatments) makes us confident that the description of the plasmonic resonances of our $\mathrm{Na}_{380}$ clusters is very reasonable. A careful review of the calculated optical properties of sodium clusters of different sizes, as well as their comparison with available experimental information, can be found in Ref. 20. One of the main conclusions that, according to our interpretation, can be obtained from examining the information presented in this reference is that A-TDLDA performs very well to describe plasmonic resonances in sodium clusters containing from a few atoms to several hundred atoms.

The $a b$ initio calculations of the atomistic models of $\mathrm{Na}_{380}$ dimers were performed using an efficient implementation of linear response TDDFT that utilizes an iterative scheme to compute the optical response in frequency domain and a local basis to expand the products of Kohn-Sham orbitals. ${ }^{3,21,22}$ The program uses as an input the information from ground-state electronic-structure calculations performed using localized basis sets (e.g., linear combination of atomic orbitals). In particular, the method has been interfaced with SIESTA and, as exemplified here, allows computing the linear optical response of large clusters and molecules with moderate computational resources. ${ }^{23}$ The results of our atomistic simulations are compared to TDDFT-LDA simulations where Na clusters are described with spherical jellium model $(\mathrm{JM})$, in which the metal is represented by an homogeneous electron gas. Only valence electrons are treated explicitly, and the ionic cores are substituted by an homogeneous background of positive charge with density $n_{+}=\left[4 \pi r_{s}^{3} / 3\right]^{-1}$. The Wigner-Zeitz radius of $\mathrm{Na} r_{s}=2.12 \AA$. Within the JM TDDFT-LDA simulations, the Kohn-Sham orbitals are represented on a real-space grid in cylindrical coordinates and their real-time evolution is explicitly calculated from the time-dependent Kohn-Sham equations. ${ }^{24}$ In this case, we have used closed shell JM spheres with 398 electrons and $15.6 \AA$ radius, i.e. similar in size to the $\mathrm{Na}_{380}$ clusters used in the atomistic calculations. Both in the atomistic and JM calculations we imposed an intrinsic (phenomenological) broadening $\Delta=300 \mathrm{meV}$, which brings the total width of the calculated plasmonic resonances in reasonable agreement with available 
experimental data. ${ }^{25}$ It is worth noting that because of the Landau damping via surface scattering ${ }^{26}$ the calculated resonances in the absorption cross-section display the full-widthat-half-maximum (FWHM) larger than the intrinsic broadening $\Delta$. Thus, in the atomistic calculations we find a $\sim 620$ meV FWHM for the relaxed clusters and in JM calculations we find a $\sim 440$ meV FWHM. The broadening obtained in the latter case is smaller because of the high spherical symmetry of the nanoparticles and the smooth boundary of the jellium sphere.

The polarizability of the atomistic $\mathrm{Na}_{380}$ model shows a well-defined plasmonic peak in the range 3.1-3.4 eV associated with a typical dipolar excitation. The computed energy of this plasmon resonance depends on the structural details and the average electron density, as well as on technical details of the calculation. In brief, the denser structure predicted by the LDA relaxation results in a slight blue shift $(\sim 150 \mathrm{meV})$ of the plasmon energy of the isolated cluster with respect to the energy obtained with the initial geometry found using empirical potentials. This blue shift is documented in Fig. S3. The primary reason for this shift is the larger average electron density (smaller volume) of the LDA-relaxed structure. The volume per atom of the initial (unrelaxed) structure is closer to the nominal one for Na. However, the LDA relaxations are important because they confirm that indeed the structure corresponds to a stable (or at least metastable) configuration of the DFT energy landscape. Moreover, a good description of the geometrical relaxations, e.g., roughness of the facets, is necessary to predict the width of the plasmon resonances. In accordance to the small geometrical changes, here the width of the plasmonic resonance only shows a small $15 \%$ increase upon relaxation.

We have carefully studied convergence of our TDDFT calculations with respect to all the technical parameters. The results show the largest dependence on the basis set of the numerical atomic orbitals used to describe the Kohn-Sham orbitals of the clusters. The DZP basis, that we choose for our calculations, contains six atomic orbitals per atom. This choice provides well-converged results around the main plasmon resonance. Namely, we 

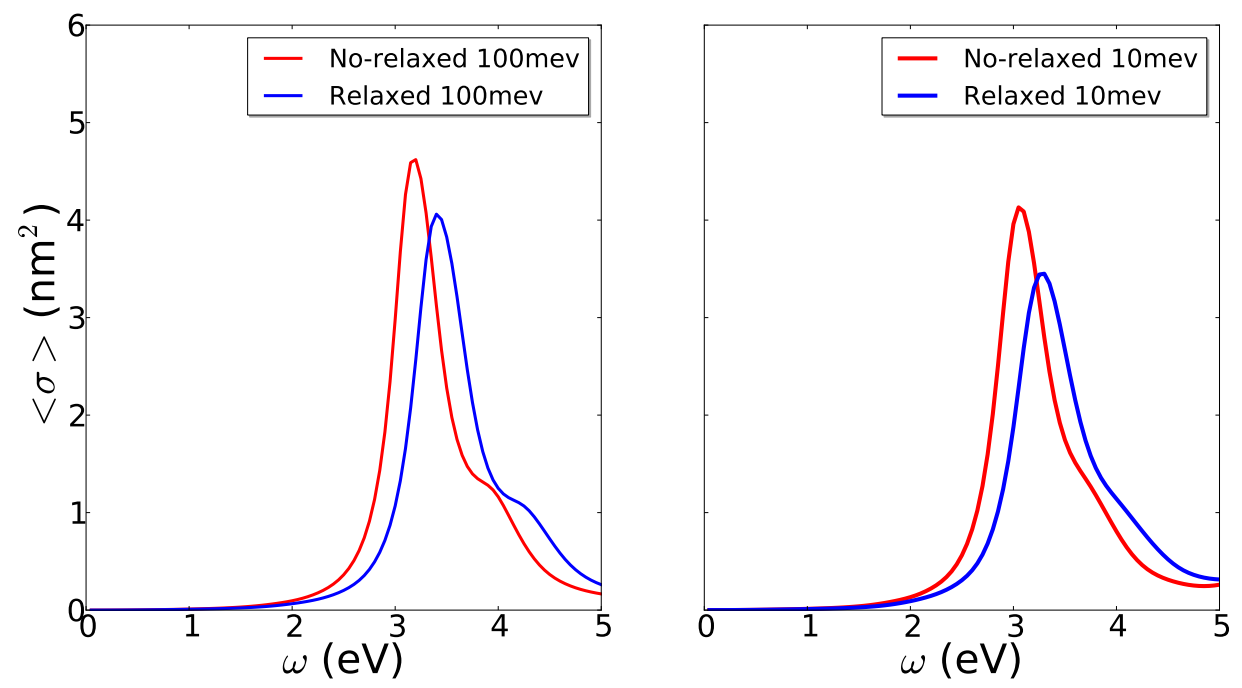

Figure S3: TDDFT-LDA results of the optical absorption of the $\mathrm{Na}_{380}$ cluster represented in Fig. S1 (averaged over all possible orientations of the exciting field). Red lines stand for result obtained for the initial structure (relaxed with empirical potentials), ${ }^{9}$ while blue lines show the results after full relaxation at the LDA level. Although both structures have an identical bonding pattern, there is a significant blue-shift of the resonance after relaxation due to the shrinkage of the structure, i.e., due to the increase of the electron density. Additionally, in left and right panels we compare results obtained with atomic orbitals with different confinement radii (details in the text of this Supp. Inf.).

have compared the DZP data with those obtained with the more complete basis set used by Tsolakidis et al. ${ }^{16}$ to study the optical response of Nas. This augmented DZP basis set has an additional shell of $d$-symmetry and thus contains eleven atomic orbitals per atom. For a fixed geometry, we get the plasmon peak of $\mathrm{Na}_{380}$ at the same position (within $10 \mathrm{meV}$ ) using these two basis (DZP and aug-DZP).

Besides the number of orbitals in the basis set, Fig. S3 also shows the dependence of the plasmon on the confinement radii $\left(\mathrm{r}_{c}\right)$ of the basis orbitals. Both the SIESTA method ${ }^{2}$ and our TDDFT code ${ }^{3}$ use basis orbitals of finite support. The shorter the confinement radius of the atomic orbitals the faster and less memory consuming the calculations. In SIESTA the radii of the orbitals are defined using the so-called energy shift parameter $\left(\mathrm{E}_{\text {shift }}\right) \cdot{ }^{7} \mathrm{We}$ have observed that the frequency of the plasmon resonance is quite sensitive to confinement of the atomic orbitals. The left panel in Fig. S3 shows the plasmon resonance obtained using 
a DZP basis set generated with $\mathrm{E}_{\text {shift }}=100 \mathrm{meV}$, corresponding to $\mathrm{r}_{c}=4.91 \AA$. This basis set provides a quite satisfactory description of the ground-state of the system. However, the relatively small extension of the orbitals tends to produce a blue shift of the plasmon frequency. This is verified in the right panel of Fig. S3, where we present the results obtained with a more extended basis $\left(\mathrm{E}_{\text {shift }}=10 \mathrm{meV}, \mathrm{r}_{c}=6.46 \AA\right)$. The plasmon resonance does not shift significantly if even more extended (smaller $\mathrm{E}_{\text {shift }}$ ) basis sets are used. As we can see in Fig. S3 there is a blue shift of approximately $100 \mathrm{meV}$ when using the $\mathrm{E}_{\text {shift }}=100 \mathrm{meV}$ with respect to the $\mathrm{E}_{\text {shift }}=10 \mathrm{meV}$ basis.

Unfortunately, the use of the $10 \mathrm{meV}$ basis sets makes calculations for the $\mathrm{Na}_{380}$ dimers quite demanding. Luckily, the trends of the modal distribution and the details of the field localization in our atomistic simulations are robust against the exact position of the reference dipolar plasmon of the isolated particle. In particular, our main result, i.e., the possibility to localize the plasmonic local fields to the atomic scale, is not significantly affected by the use of the $100 \mathrm{meV}$ versus the $10 \mathrm{meV}_{\text {shift }}$ basis. Therefore, in order to alleviate the computational task and allow considering several dimer configurations and many different inter-cluster distances, we have decided to use the orbitals obtained with $\mathrm{E}_{\text {shift }}=100 \mathrm{meV}$.

The JM results are obtained with a completely independent framework ${ }^{24}$ and thus the calculations are not affected by these uncertainties. The positive background charge density is fixed to the nominal one of bulk Na. The wave-functions are discretized in a two dimensional grid exploiting the cylindrical symmetry of the problem. This reduces considerable the computational effort, allowing to converge the results with respect to the fineness of the grid.

We compare the optical response of the JM and the atomistic results (LDA-relaxed structures) for an isolated cluster and a dimer with $\mathrm{d}_{\mathrm{sep}}=10 \AA$ in Fig. S4. Notice that, for the atomistic calculations, we cannot easily read the red shift of the plasmon due to the interaction in the dimer. This is because we are plotting different responses in each case: for the isolated cluster the response is averaged over all possible field directions, while for the 

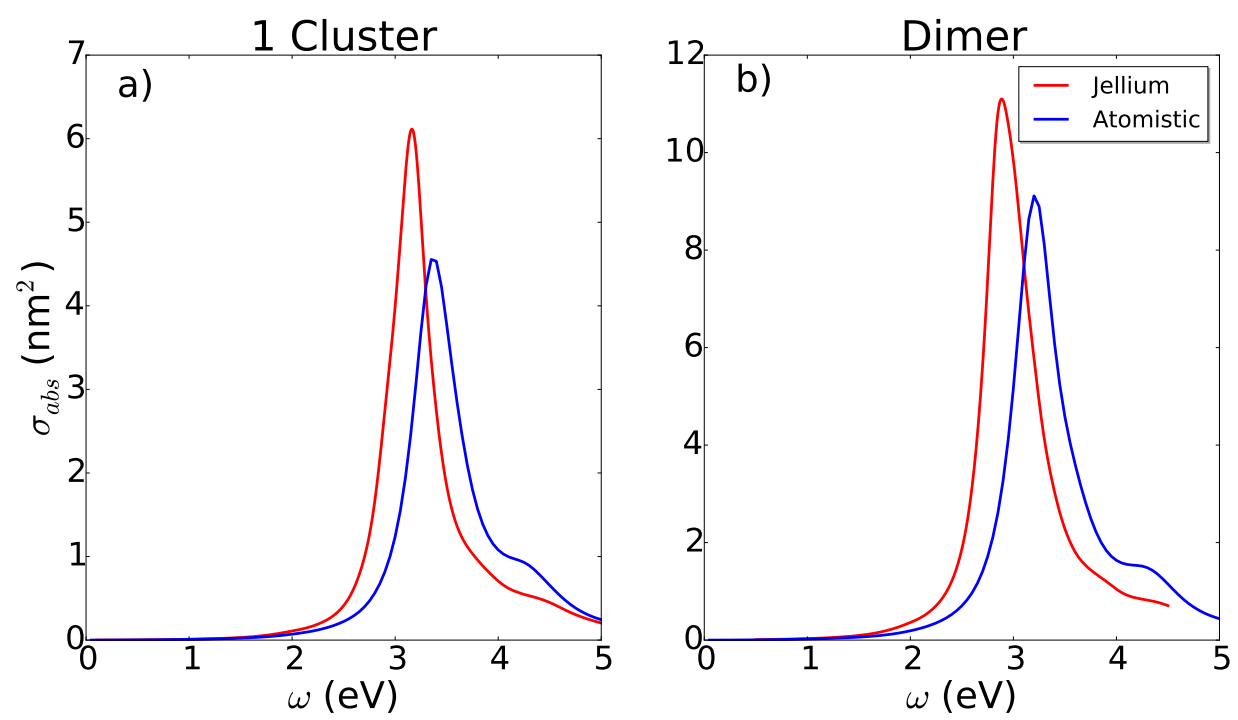

Figure S4: Comparison of the optical absorption cross-section calculated using TDDFT-LDA for a spherical jellium cluster (398 electrons, $\sim 15.6 \AA$ radius, $\mathrm{r}_{s}=2.12 \AA$ ) and the $\mathrm{Na}_{380}$ cluster in Fig. S1. Panel a) shows the comparison for the a single cluster (absorption averaged over all directions of the incident field), while panel b) compares data for dimers with $d_{\text {sep }}=10 \AA$ and the polarization of the incident field along the dimer-axis (the atomistic data correspond to the facet-to-facet case).

dimer we only consider the response for a field oriented along the dimer axis. However, the expected red shift exists, as discussed later in detail and apparent in Fig. S5 and Fig. 2 of the main text of the letter. Besides the higher energy of the plasmon resonance in the atomistic results, which is mainly due to the higher electron density in the LDA-relaxed cluster, the width of the plasmon resonance of the isolated cluster is smaller in the JM case. For the JM the FWHMs are 440 and 510 meV, respectively, for the isolated cluster and the $\mathrm{d}_{\text {sep }}=10 \AA$ dimer. In the atomistic calculations those widths increase respectively to 620 and $570 \mathrm{meV}$. In the atomistic calculations of the isolated cluster, part of the additional broadening comes from averaging over inequivalent field-polarization directions. This inhomogeneous broadening mechanism accounts for $\sim 80 \mathrm{meV}$ of the total width. Still, the atomistic calculation predicts a plasmon resonance $\sim 100 \mathrm{meV}$ broader than the JM for the isolated cluster. The enhanced electron-hole scattering ${ }^{26}$ at sharp edges, as well as, at defects and roughness of the surfaces are probably responsible of this extra-broadening. These factors are known to 
broaden the plasmon resonances for sufficiently small clusters, when the cluster dimensions are smaller or comparable to the mean free path of the electrons. ${ }^{27,28}$ For the dimer both calculations report a closer width, although still it is clearly larger in the atomistic case.

\section{Evolution of the absorption cross-section of the $\mathbf{N a}_{380}$ dimer as a function of $d_{\text {sep }}$}

Here we discuss in detail the evolution of the optical spectrum of the dimers as a function of the nanogap size $d_{\text {sep }}$. In Fig. S5 (also in Fig. 2 of the main text of the letter) we can distinguish the evolution of the hybridized Bonding Dimer Plasmon (BDP) that redshifts (lowers the resonance energy) as the nanoparticles of the dimer become closer. Consistent with previous descriptions, when the particles are very close together, at a separation of a few Angstroms, the BDP is screened due to the emergence of the tunneling current at optical frequencies..$^{24,29,30}$ The three atomistic cases show a similar qualitative tendency for this screening, but remarkable differences arise with regard to the exact separation distance where this quantum effect occurs. For the facet-to-facet nanogap [Fig. S5 a)], the screening

of the BDP takes place at a separation, $d_{\text {sep }}$, of about 6 Angstroms, which is the largest among the configurations studied here. In Fig. S5 a) we can also see the emergence of a charge transfer plasmon $(\mathrm{CTP})^{31}$ at a separation distance of about $4 \AA$. The CTP mode involves the polarization of the whole connected dimer, and appears as a consequence of the tunneling current established across the gap. Substantial differences in the behavior of the BDP and CTP can be identified for the tip-to-facet and tip-to-tip situations [Fig. S5 b) and c) respectively]. When a tip-like end is present in one of the interfaces, the tunneling across the gap is reduced since it is mediated mainly through the tip protrusion, and therefore, the screening of the BDP is not so evident. Instead we observe a clear transfer of the spectral weight towards the higher energy quadrupolar (QP) mode. ${ }^{32}$ This modal redistribution is much weaker in the facet-to-facet case. The emergence of the CTP, and the complete 

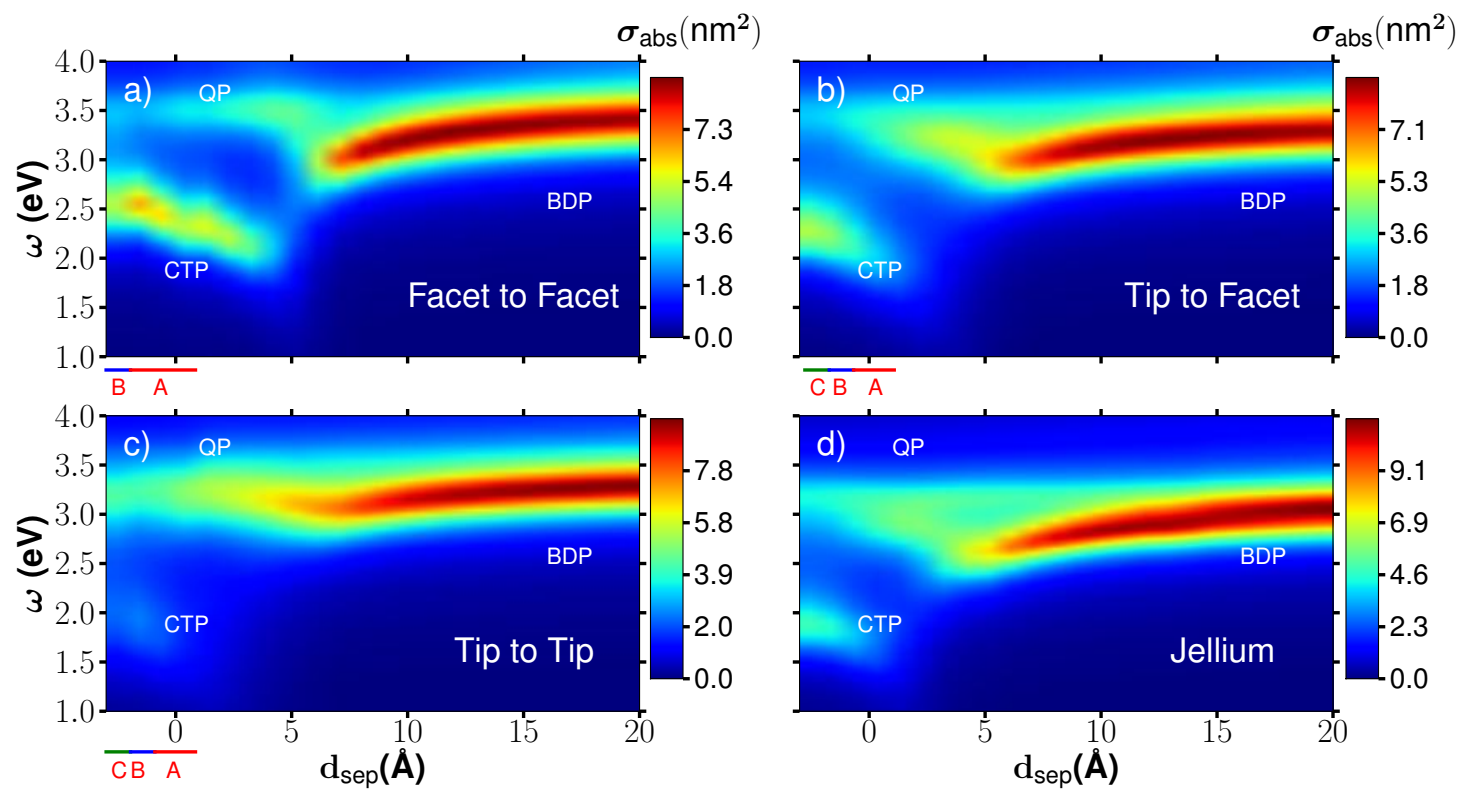

Figure S5: Panels (a)-(d) show the spectral evolution of the absorption cross-section for different plasmonic dimers as a function of separation distance between the particles $d_{\text {sep }}$. The incident field is polarized along the dimer axis. Panels show results for the facet-to-facet dimer a), tip-to-facet b), tip-to-tip c) and the jellium sphere d) dimers. These data are also shown in Fig. 2 of the main text of this letter and are repeated here only for convenience of the reader. The hybridized bonding dipolar plasmon (BDP) is red shifted as the separation distance decreases, and it is finally screened due to the emergence of tunneling between the particles. A charge-transfer plasmon (CTP) mode emerges as the particles come into contact or due to the tunneling current. Each of the configurations shows different features depending on the morphology. Distances lower than $1 \AA$ and negative distances represent overlapping clusters ${ }^{11}$ and have been modeled using the geometries described in Fig. S2 and the corresponding text. As expected due to the shortening of the structure, the dipolar CTP is blueshifted as the "overlap" between the clusters increases.

quenching of the higher energy BDP and QP modes, is postponed to a separation of about 1 $\AA$ [Fig. S5 b)]. This situation is even more pronounced in the case of a tip-to-tip geometry, where the emergence of the CTP is postponed until both particles overlap [see Fig. S5 c)]. A dimer described within the JM also shows the quenching of the BDP and the emergence of the CTP. In this case, the gap between ideal spherical surfaces results in an optical spectrum similar to an intermediate situation between the tip-to-facet and facet-to-facet geometries. Thus the modal redistribution also occurs for intermediate separation distances $(\sim 3 \AA)$ in the JM. 

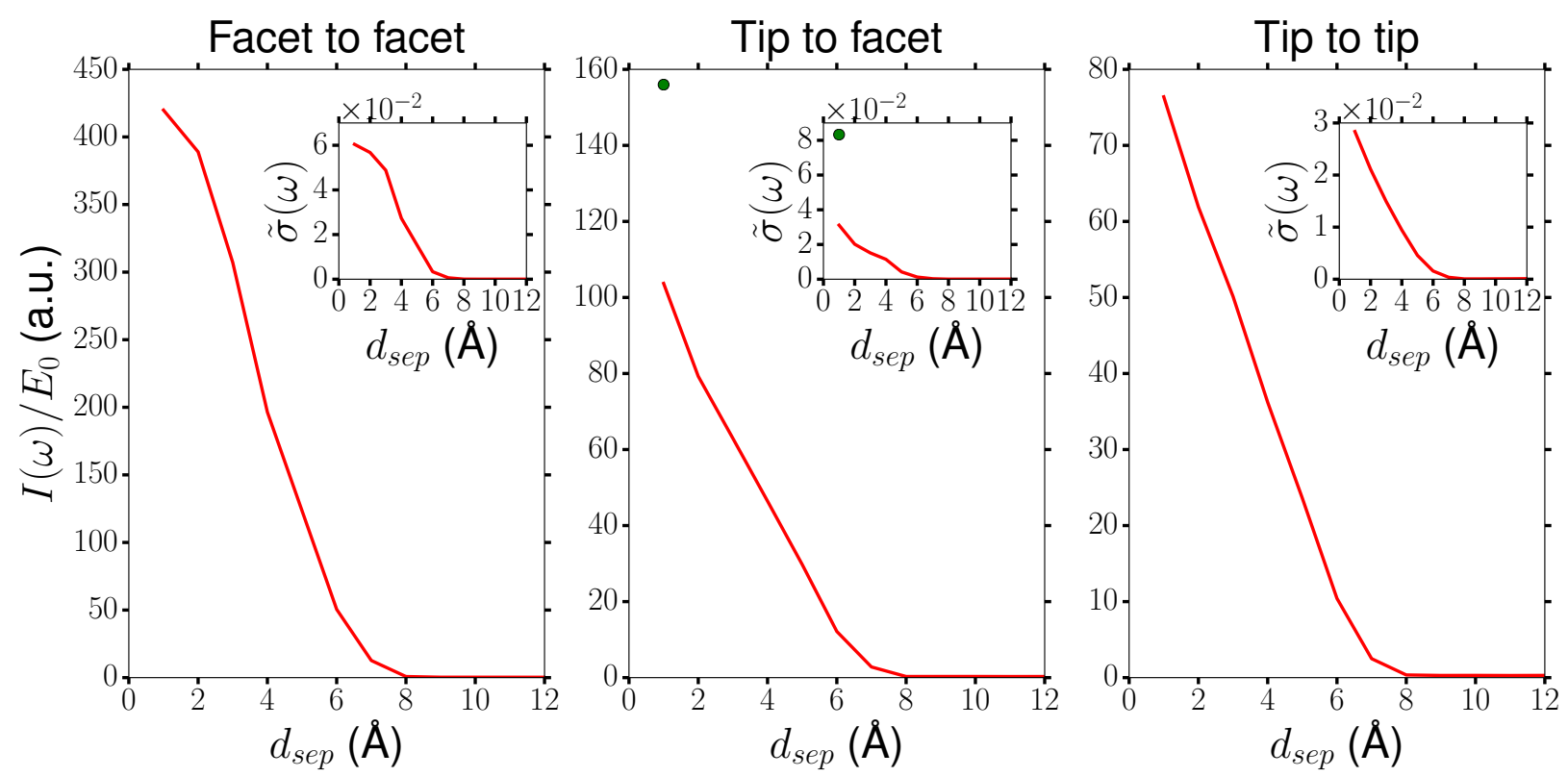

Figure S6: Amplitude of the electron current (in atomic units and normalized by the external field) flowing between the two $\mathrm{Na}_{380}$ clusters as a function of the interparticle separation $d_{\text {sep }}$ for the three plasmonic gaps considered here. The continuous lines represent the data computed at the frequencies of the most intense resonances of the absorption cross-section shown in Fig. S5. At sufficiently large distances this corresponds to the BDP mode for the three configurations. For the facet-to-facet case at $d_{\text {sep }}<5 \AA$ we plot the current associated with the CTP mode, while for the tip-to-facet and tip-to-tip gaps we follow the QP mode around $3 \mathrm{eV}$ at small values of $\mathrm{d}_{\mathrm{sep}}$. In the tip-to-facet case we also show for comparison (filled circle) the current computed for $\omega \sim 1.9 \mathrm{eV}$ at $d_{\text {sep }}=1 \AA$, i.e., at the onset of the CTP mode for this configuration. The insets of each panel show an estimation of the conductivity, $\tilde{\sigma}$, as a function of separation distance for each configuration considered. This conductivity is obtained as a ratio between the effective current density, and the local field at the gap, as described in the text of this Supp. Inf.

The link between the appearance of the CTP mode (and the quenching of the BDP mode) and the increase of the electron current flowing through the gap can be clearly established with help of Fig. S6. In this figure we present the amplitude of the electron current divided by the external field as a function of the interparticle separation $d_{\text {sep }}$. The currents are computed at the frequencies of the most intense resonances of the absorption cross-section (see Fig. S5) for each distance. At sufficiently large distances this frequency always corresponds to the BDP mode for the three configurations. However, for $d_{\text {sep }}<5 \AA$ we plot the current associated with the CTP mode for the facet-to-facet case, while for the tip-to- 
facet and tip-to-tip gaps we follow the QP mode around $3 \mathrm{eV}$ for those small values of $d_{\text {sep }}$. The currents are calculated, using the induced electron-density at a given frequency, as the charge crossing a surface bisecting the gap by unit of time. As expected, in all cases the current grows steeply as $d_{\text {sep }}$ is reduced. The strongest currents are obtained at the frequencies and distances where a well-defined CTP mode appears (left panel for the facet-to-facet configuration, and filled circle in the middle panel for the tip-to-facet configuration). For example, at small $d_{\text {sep }}$ the current is approximately four times larger for the CTP mode in the facet-to-facet case than that computed for the QP mode in the tip-to-facet geometry. In the tip-to-facet case we also show (filled circle) for comparison the current computed at $\omega \sim 1.9 \mathrm{eV}$ and $d_{\mathrm{sep}}=1 \AA$, i.e., at the onset of the CTP mode. It is a $\sim 50 \%$ larger than the current for QP mode at the same distance. This clearly indicates that, for the same geometry and gap separation, the current is considerably larger in the CTP mode than in the QP mode. It is also interesting to note that the current computed at the onset of the CTP for the tip-to-facet configuration is almost identical to the current computed at the onset of the CTP mode for the facet-to-facet case (although $d_{\text {sep }}$ is in the range $4-5 \AA$ in the latter case and $1 \AA$ in the former). This is to be expected, since the establishment of the CTP mode depends on the possibility of charge to be transferred through the gap. Another interesting conclusion from our analysis is that an atomic contact formed by a single $\mathrm{Na}$ atom (like in our "tip configurations") is insufficient to sustain the current flow necessary for the appearance of a fully developed CTP mode, consistent with the results of Fig. S5.

Finally, we also show in the insets of Fig. S6 an estimation of the conductivity, $\tilde{\sigma}$, as a function of separation distance for each configuration considered. This conductivity is obtained as a ratio between the effective current density, and the local field at the gap. The effective current density is obtained by dividing the current intensity $I(\omega)$ by the minimum of the localization area of Fig. $3 \mathrm{~b}$ ), $\mathcal{A}$, of the main text, and the local field is estimated by multiplying the external field $E_{0}$ by the local field enhancement obtained in Fig. 3 a), $\left|\mathbf{E}_{\text {ind }}^{\max }\right| / E_{0}$, of the main text. The currents and conductivities estimated in this way for 
the facet-to-facet configuration (inset of the left panel in Fig. S6) are consistent with those recently reported between two large jellium spheres representing Na clusters. ${ }^{33}$

Coming back to the discussion of Fig. S5, distances lower than $1 \AA$ and negative distances represent overlapping clusters. This type of configurations have been extensively studied using classical electromagnetic simulations. ${ }^{11}$ The structures utilized to describe this overlapping situation are presented in panels A, B and C of Fig. S2 and are obtained by subsequently removing one atomic layer from one of the clusters forming the gap (up to three layers for the "tip-configurations"). Remarkably the plasmon resonances and the intensity of the optical absorption changes quite smoothly as we move from configurations type-A, to type-B and C. As can be expected, for the facet-to-facet nanogap the main effect is the blue-shift (and a slight intensity increase) of the CTP resonance as the total length of the connected structure decreases with the coalescence of the clusters. ${ }^{11}$ In the case of the tip-tofacet nanogap, not only the length of the united-dimer decreases, but the cross-section of the junction increases as we move from configurations type-A to C. As a consequence, the emergence of a well-developed CTP mode only takes place when the geometrical cross-section is sufficiently large, and correspondingly the conductance of the junction. Thus, the CTP is clearly observed for configuration type-B and $\mathrm{C}$, while type-A dimer produces only a very faint CTP signal as explained above. This is even more clear in the tip-to-tip case, where the cross-section of the junction is determined by the tip left intact in one of the clusters when the atomic layers are removed from the other. Due to this geometry-construction procedure, we always have a one-atom cross-section and a strong CTP mode is never observed for all three A, B and C-type configurations.

\section{References}

(1) Sánchez-Portal, D.; ; Ordejón, P.; Artacho, E.; Soler, J. M. Int. J. Quantum Chem. 1997, 65, 453-461. 
(2) Soler, J. M.; Artacho, E.; Gale, J. D.; García, A.; Junquera, J.; Ordejón, P.; SánchezPortal, D. J. Phys.: Condens. Matter 2002, 14, 2745-2779.

(3) Koval, P.; Foerster, D.; Coulaud, O. J. Chem. Theory Comput. 2010, 6, 2654-2668.

(4) Kohn, W.; Sham, L. J. Phys. Rev. 1965, 140, A1133-A1138.

(5) Perdew, J. P.; Zunger, A. Phys. Rev. B 1981, 23, 5048-5079.

(6) Troullier, N.; Martins, J. L. Phys. Rev. B 1991, 43, 1993-2006.

(7) Artacho, E.; Sánchez-Portal, D.; Ordejón, P.; García, A.; Soler, J. M. Phys. Status Solidi B 1999, 215, 809-817.

(8) Murrell, J. N.; Mottram, R. E. Mol. Phys. 1990, 69, 571-585.

(9) The initial structure of the $\mathrm{Na}_{380}$ cluster was download from the Cambridge Cluster Database, D. J. Wales, J. P. K. Doye, A. Dullweber, M. P. Hodges, F. Y. Naumkin, F. Calvo, J. Hernández-Rojas and T. F. Middleton, URL http://wwwwales.ch.cam.ac.uk/CCD.html.

(10) Cohen, M. L.; Dacorogna, M. M. Phys. Rev. B 1986, 34, 4996-5002.

(11) Romero, I.; Aizpurua, J.; Bryant, G. W.; García de Abajo, F. J. Opt. Express 2006, 14, 9988-9999.

(12) Runge, E.; Grosss, E. K. U. Phys. Rev. Lett. 1984, 52, 997-1000.

(13) Rubio, A.; Alonso, J. A.; Blase, X.; Balbás, L. C.; Louie, S. G. Phys. Rev. Lett. 1996, rॅ, $247-250$.

(14) Vasiliev, I.; Ögüt, S.; Chelikowsky, J. R. Phys. Rev. Lett. 1999, 82, 1919-1922.

(15) Vasiliev, I.; Öğüt, S.; Chelikowsky, J. R. Phys. Rev. B 2002, 65, 115416.

(16) Tsolakidis, A.; Sánchez-Portal, D.; Martin, R. M. Phys. Rev. B 2002, 66, 235416. 
(17) Bonačić-Koutecký, V.; Fantucci, P.; Koutecký, J. J. Chem. Phys. 1990, 93, 3802-3825.

(18) Bonačić-Koutecký, V.; Fantucci, P.; Koutecký, J. Chem. Phys. Lett. 1990, 166, 32-38.

(19) Marques, M. A. L., Ulrich, C. A., Nogueira, F., Rubio, A., Burke, K., Gross, E. K. U., Eds. Time-Dependent Functional Theory; Lecture Notes in Physics 706; Springer, Berlin Heidelberg, 2006.

(20) Alonso, J. A. Structure and Properties of Atomic Nanoclusters; NanoScience and Technology; Imperial College Press, 2006.

(21) Koval, P.; Foerster, D.; Coulaud, O. Phys. Status Solidi B 2010, 247, 1841-1848.

(22) Foerster, D.; Koval, P. J. Chem. Phys. 2009, 131, 044103.

(23) Manjavacas, A.; Marchesin, F.; Thongrattanasiri, S.; Koval, P.; Nordlander, P.; Sánchez-Portal, D.; García de Abajo, F. J. ACS Nano 2013, 7, 3635-3643.

(24) Marinica, D. C.; Kazansky, A. K.; Nordlander, P.; Aizpurua, J.; Borisov, A. G. Nano Lett. 2012, 12, 1333-1339.

(25) Schmidt, M.; Haberland, H. Eur. Phys. J. D 1999, 6, 109-118.

(26) Yannouleas, C.; Broglia, R. A. Ann. Phys. 1992, 217, 105-141.

(27) Prodan, E.; Nordlander, P.; Halas, N. J. Nano Lett. 2003, 3, 1411-1415.

(28) Kreibing, U.; Vollmer, M. Optical Properties of Metal Clusters; Springer, New York, 1995.

(29) Zuloaga, J.; Prodan, E.; Nordlander, P. Nano Lett. 2009, 9, 887-891.

(30) Esteban, R.; Borisov, A. G.; Nordlander, P.; Aizpurua, J. Nat. Commun. 2012, 3, 825.

(31) Pérez-González, O.; Zabala, N.; Borisov, A. G.; Halas, N. J.; Nordlander, P.; Aizpurua, J. Nano Lett. 2010, 10, 3090-3095. 
(32) Marinica, C.; Lourenço-Martins, H.; Aizpurua, J.; Borisov, A. G. Nano Lett. 2013, 13, 5972-5978.

(33) Esteban, R.; Zugarramurdi, A.; Zhang, P.; Nordlander, P.; García-Vidal, F. J.; Borisov, A. G.; Aizpurua, J. Faraday Discuss. DOI: 10.1039/C4FD00196F. 